\title{
Exponential growth of torsion in abelian coverings
}

\author{
JEAN RAIMBAULT
}

\begin{abstract}
We show exponential growth of torsion numbers for links whose first nonzero Alexander polynomial has positive logarithmic Mahler measure. This extends a theorem of Silver and Williams to the case of a null first Alexander polynomial and provides a partial solution for a conjecture of theirs.
\end{abstract}

57M10; 57M25, 57Q10

\section{Introduction}

Let $M$ be a compact three-manifold; the homology groups $H_{i}(M)$ can be written as the direct sums $H_{i}(M)_{\text {tors }} \oplus H_{i}(M)_{\text {free }}$ of a finite abelian group with a finite-rank free abelian group. The torsion summand is nontrivial only for $i=1: H_{0}$ and $H_{3}$ are $\mathbb{Z}$ or 0 , and the universal coefficients theorem for cohomology implies that $H^{1}(M, \partial M)$ is free, and by Poincaré duality it follows that $\mathrm{H}_{2}(M)$ is also torsion-free. On the other hand, the torsion in $H_{1}$ can be arbitrarily large (for example, for lens spaces; see below for hyperbolic examples) and it is believed that "most" 3-manifolds should have a rather large torsion. For example E Kowalski shows in [9, Proposition 7.19] that the first homology group of a "Dunfield-Thurston random 3-manifold" typically has a large torsion subgroup. This paper is concerned with the growth rate of the order of $H_{1}\left(M_{N}\right)_{\text {tors }}$ in a sequence of finite coverings $M_{N}$ of a manifold $M$. The least precise question that can be asked is whether it is exponential in the degree or not, that is, whether the sequence $\frac{\log \left|H_{1}\left(M_{N}\right)_{\text {tors }}\right|}{\left[\pi_{1}(M): \pi_{1}\left(M_{N}\right)\right]}$ has a positive limit (or limit superior). This shall be partially answered here in the case where the $M_{N}$ are abelian coverings converging to a free abelian covering of $M$. The main motivation to study this question was to provide a partial result towards a conjecture of Silver and Williams on the growth rate of torsion numbers of abelian coverings of complements of links (see Silver-Williams [24, Conjecture 6.1] or (0-1) below).

Historically, the first context where the growth of torsion in the homology of coverings has been studied is that of cyclic coverings of a knot complement. Let $K$ be an open knotted solid torus in the three-sphere and $M=\mathbb{S}^{3}-K$; then $M$ is a compact threemanifold with $H_{1}(M)=\mathbb{Z}$. Thus we can consider the infinite cyclic covering $\widehat{M}$ of $M$ and its finite quotients $M_{N}$, which are the finite coverings of $M$ corresponding to 
the maps $\pi_{1}(M) \rightarrow \mathbb{Z} \rightarrow \mathbb{Z} / N \mathbb{Z}$. If $\Delta$ is the Alexander polynomial of $K$ (the first Alexander polynomial of the $\mathbb{Z}[\mathbb{Z}]$-module $H_{1}(\widehat{M})$, see 1.2$)$ then the so-called Fox formula says that for all $N$ such that $H_{1}\left(M_{N}\right)$ has rank one we have

$$
\left|H_{1}\left(M_{N}\right)_{\text {tors }}\right|=\prod_{\zeta^{N}=1}|\Delta(\zeta)| .
$$

It is known that $\Delta$ is always nonzero. A diophantine inequality due to Gelfond then allows to show that when $N$ tends to infinity $\frac{1}{N} \sum_{\zeta^{N}=1} \log |\Delta(\zeta)|$ converges to the logarithmic Mahler measure of $\Delta$, that is,

$$
m(\Delta)=\int_{\mathbb{T}^{1}} \log |\Delta(z)| d z
$$

(here $\mathbb{T}^{1}$ is the unit circle in $\mathbb{C}$ endowed with the Lebesgue probability measure); see Lemma 2.6 below or Silver-Williams [25, Proposition 2.8]. Let $d$ be the product of all integers $l$ such that the $l$ th cyclotomic polynomial divides $\Delta$ : it is known (see SilverWilliams [25, Proposition 2.2] or Gordon [6, Theorem 4.1(ii)]) that $H_{1}\left(M_{N}, \mathbb{Q}\right) \cong$ $H_{1}\left(M_{N+d}, \mathbb{Q}\right)$. We get that

$$
\lim _{\substack{N \rightarrow \infty \\ d \mid N-1}}\left(\frac{1}{N} \log \left|H_{1}\left(M_{N}\right)_{\text {tors }}\right|\right)=m(\Delta)
$$

in particular, if $m(\Delta)>0$ an infinite subsequence of the torsion numbers tends to infinity with exponential growth. This result answered a question of Gordon (who proved in [6] that when $m(\Delta)=0$ the torsion numbers are periodic in $N$ ) and has first been proved by González-Acuña and Short in [5] and independently by Robert Riley in [20]. Since there exists hyperbolic knots whose Alexander polynomial has positive Mahler measure (for example the figure-eight) this proves in particular that there exists (noncompact with finite volume) hyperbolic manifolds with arbitrarily large torsion in their $H_{1}$.

The similar setting for a collection of $m>1$ linked solid tori $L$ in the three-sphere is to consider the coverings $M_{H}$ of $M=\mathbb{S}^{3}-L$ given by the maps $\pi_{1}(M) \rightarrow H_{1}(M) \cong$ $\mathbb{Z}^{m} \rightarrow \mathbb{Z}^{m} / H$ where $H$ is a finite-index subgroup. We want to study the torsion numbers as the quantity

$$
\alpha(H)=\min \left\{\max _{i}\left|v_{i}\right|: 0 \neq v=\left(v_{1}, \ldots, v_{m}\right) \in H\right\}
$$

tends to infinity. Fox's formula admits a generalization due to Mayberry and Murasugi [18]; see also Porti [19] for a proof using Reidemeister torsion. However, because the Betti numbers may very well tend to infinity as $\alpha(H)$ does, it may not be applicable to an infinite sequence of coverings. This forbids any naïve application of the preceding 
scheme of proof to this case. Moreover, two other obstructions arise when considering links: the Alexander polynomial may be zero, and the generalisation of Gelfond's estimate needed to prove the convergence of Riemann sums to the Mahler measure is not known to hold for polynomials in several variables.

By using methods from algebraic dynamical systems Daniel Silver and Susan Williams, in [24], were able to show that if the first Alexander polynomial $\Delta(L)$ of $L$ is nonzero then

$$
\limsup _{\alpha(H) \rightarrow \infty} \frac{\log \left|H_{1}\left(M_{H}\right)_{\text {tors }}\right|}{[G: H]}=m(\Delta(L)):=\int_{\mathbb{T}^{m}} \log |\Delta(L)|
$$

and that this holds with a limit in the case of a knot (this is also proved in [25]). They also conjecture that, in the general case, the following limit should hold where $\Delta_{i}$ is the first nonzero polynomial in the sequence of Alexander polynomials of $L$ :

$$
\lim _{\alpha(H) \rightarrow \infty} \frac{\log \left|H_{1}\left(M_{H}\right)_{\text {tors }}\right|}{[G: H]}=m\left(\Delta_{i}(L)\right)
$$

The Mahler measure of the Alexander polynomial of a knot can be interpreted as the $\ell^{2}$-torsion of the infinite cyclic covering $\widehat{M}$ of its exterior $M$ (see for example (1-5) below). In [1] Nicolas Bergeron and Akshay Venkatesh used this to give a new proof of the theorem of Silver and Williams in the case of a knot: the main point is that the nonnullity of the Alexander polynomial guarantees that $\widehat{M}$ is $\ell^{2}$-acyclic, and in this case the approximation of $\ell^{2}$-torsion by Reidemeister torsions is known. They then show that the growth of torsion numbers is the same as that of Reidemeister torsion and obtain a generalization of the theorem for knots (see [1, Theorem 7.3]). For links with nonzero first Alexander polynomial their proof can be adapted; however the result obtained is slightly weaker than Silver and Williams'.

The aim of this work is to generalise their results to the case of a null first Alexander polynomial, that is, that of a non- $\ell^{2}$-acyclic infinite covering. This yields the following result, which follows from the more general Theorem 3.1.

Theorem 0.1 With notation as in (0-1) we have

$$
\limsup _{\alpha(H) \rightarrow \infty} \frac{\log \left|H_{1}\left(M_{H}\right)_{\text {tors }}\right|}{[G: H]} \geq m\left(\Delta_{i}(L)\right) .
$$

The same scheme of proof can be applied to homology with coefficients in a local system. Let $V$ be a free $\mathbb{Z}$-module of finite rank and $\chi$ a representation of $\pi_{1}(M)$ to $G L(V)$. Then we can define homology twisted by $\chi$ for all coverings of $M$, also called homology with coefficients in the local system defined by $\chi$. See Hatcher [7, 
Chapter 3.H] for definitions. Let $\Delta_{i}(L, \chi)$ be the first nonzero Alexander polynomial of $H_{1}(\widehat{M} ; V)$ (the Alexander polynomials with nontrivial coefficients are usually called twisted Alexander polynomials). We get (see also Silver-Williams [26, Theorem 3.10])

$$
\limsup _{\alpha(H) \rightarrow \infty} \frac{\log \left|H_{1}\left(M_{H} ; V\right)_{\text {tors }}\right|}{[G: H]} \geq m\left(\Delta_{i}(L, \chi)\right) .
$$

In the case of a sequence of cyclic coverings converging to a possibly non- $\ell^{2}$-acyclic infinite cyclic covering the proofs yield a more precise result, generalising [25, Theorem 2.10].

Theorem 0.2 If $M$ is a compact $n$-manifold (with boundary) with a surjection $\pi_{1}(M) \rightarrow \mathbb{Z}$ let $M_{N}$ be the cyclic covering of $M$ associated to $\pi_{1}(M) \rightarrow \mathbb{Z} / N \mathbb{Z}$ and $\widehat{M}$ the infinite cyclic covering. Then we have for all $i=1, \ldots, n-1$ :

$$
\lim _{N \rightarrow \infty} \frac{\log \left|H_{i}\left(M_{N}\right)_{\text {tors }}\right|}{N}=m\left(\Delta_{i}\right)
$$

where $\Delta_{i}$ is the first nonzero Alexander polynomial of $H_{i}(\widehat{M})$.

Thang Le has recently proved the equality lacking in Theorem 0.1 in the preprint [13]. His proof uses methods slightly different from ours, namely a Bourbaki theorem on "pseudo-zero modules" and the dynamical version of approximation (due to Lind; see Schmidt [22, Theorem 21.1]). He also gives a different construction of the approximating sequences constructed in Proposition 2.7 below.

Finally, note that throughout this paper we work with three-manifolds with boundary, that is, we consider regular coverings of $\mathbb{S}^{3}-L$ instead of the corresponding branched (over core circles of $L$ ) coverings of $\mathbb{S}^{3}$, which are closed three-manifolds. However, the growth of torsion homology in abelian coverings is the same for the two sequences of manifolds, as shown in Le [13,4.4] (for knots the two homologies differ only by a infinite cyclic direct factor).

\section{Warnings and outline}

Most of the techniques and results used here are fairly elementary with one notable exception: I have found more practical and efficient to use the Fuglede-Kadison determinant as defined in the book [17] of Wolfgang Lück, and all its properties proved therein instead of trying to do everything solely in terms of Mahler measures (in any case, it is the right tool to use to define $\ell^{2}$-torsion). However, the reader need not be familiar with the Fuglede-Kadison determinant since all those of its properties that we use are recalled. One warning about notations: I have chosen to denote by $\mathcal{M}$ the 
multiplicative Mahler measure given by $\exp (m)$ since I already use the casual $M$ to denote modules and manifolds.

The paper is organised as follows: the first section reviews various classical and $\ell^{2}$-invariants for $\mathbb{Z}[G]$-modules. In particular, I give there the computation of the $\ell^{2}$-torsion in terms of Mahler measures of Alexander polynomials. In the second section I give a proof of the approximation of the Fuglede-Kadison determinant in the free Abelian case since there was (to the best of my knowledge) no complete proof of this result available in the literature. In the third section we prove Theorems 0.1 and 0.2. The fourth section contains some further remarks about three-manifolds. The appendices contain proofs that I judged too cumbersome for a first reading; I hope that their being relegated there will make the main thrust of the paper more apparent and intelligible.

\section{Acknowledgements}

The subject treated here has been proposed to me by my PhD advisor, Nicolas Bergeron. I gratefully thank him for this as well as for the numerous suggestions he made regarding the redaction of this paper and the math inside. I also wish to thank Pierre Vogel who tried to explain to me the Alexander polynomial. I have benefited from conversations with Thang Le regarding his paper [13] and related topics, as well as his pointing out a deadly mistake in a previous proof of Theorem 3.7. Also, the Bombieri-Zannier theorem used in Appendix A has been brought to my attention by the notes from a lecture he gave. Finally, I thank Takahiro Kitayama for reading carefully a previous version of this paper and the referee for spotting many typos and minor flaws.

\section{Review of classical and $\ell^{2}$-invariants}

In this section $G$ shall denote a free Abelian group of rank $m$; we identify the group ring $\mathbb{Z}[G]$ with the Laurent polynomials $\mathbb{Z}\left[t_{1}^{ \pm 1}, \ldots, t_{m}^{ \pm 1}\right]$ through the choice of a free generating set $t_{1}, \ldots, t_{m}$ for $G$. In this section, we review various invariants for $G$-modules and $G$-complexes.

\subsection{Rank, von Neumann dimension and $\ell^{2}$-Betti numbers}

Recall that a finitely generated Hilbert $\mathcal{N}(G)$-module is a Hilbert space with a unitary action of $G$, which admits a $G$-equivariant surjection of $\ell^{2}(G)^{n}$ for some integer $n$ (we shall not use explicitly the von Neumann algebra $\mathcal{N}(G)$ in the sequel, so we shall simply call these finitely generated $\mathcal{N}(G)$-modules). Given a submodule $M \subset \mathbb{Z}[G]^{n}$, 
the closure of $M \otimes \mathbb{C}$ is a $\mathcal{N}(G)$-submodule of $\ell^{2}(G)^{n}$, in particular it is a finitely generated $\mathcal{N}(G)$-module. The von Neumann dimension of a $\mathcal{N}(G)$-module is defined for any discrete group (see Lück [17, Definition 1.10]): it assigns to a nonzero finitely generated $\mathcal{N}(G)$-module a positive real number and satisfies the same basic properties that the dimension of a vector space does (see [17, Theorem 1.12]). In general it is not easy to compute. However, in our case the group ring $\mathbb{Z}[G]$ has a fraction field $\mathbb{Q}(G)$ identified with the field of rational functions in $m$ variables $\mathbb{Q}\left(t_{1}, \ldots, t_{m}\right)$. We can then define the rank of $M$ as the dimension of the $\mathbb{Q}(G)$-vector space $M \otimes \mathbb{Q}(G)$.

Lemma 1.1 Let $M$ be a submodule of $\mathbb{C}[G]^{n}$; then the von Neumann dimension of its closure in $\ell^{2}(G)^{n}$ is equal to its rank.

Proof This is easy to deduce from the two lemmas below: according to Lemma 1.2 we can pick a free module $L \subset M$ such that $M / L$ is torsion; then the rank of $M$ is equal to that of $L$, and by Lemma 1.3 the completion of $L$ is equal to that of $M$. Thus it suffices to show the lemma for a free submodule $L$. Let $r$ be the rank of $L$; then $L$ is the image of an injective morphism from $\mathbb{C}[G]^{r}$ to $\mathbb{C}[G]^{n}$, which extends to an isomorphism of $\mathcal{N}(G)$-modules from $\ell^{2}(G)^{r}$ to the closure of $L$, so that its von Neumann dimension is $r$.

Lemma 1.2 Let $M$ be a finitely generated $\mathbb{Z}[G]$-module. If $L \subset M$ is a maximal rank free submodule (which always exists) then the quotient $M / L$ is torsion.

Proof If $a_{1}, \ldots, a_{r}$ is a basis for the finite-dimensional vector space $M \otimes \mathbb{C}(G)$ such that $a_{i} \in M$ for all $i$, then the module $L=\sum_{i} \mathbb{Z}[G] a_{i}$ is a maximal rank free submodule. If $f$ is the lowest common multiple of the denominators of the coordinates of a generating family for $M$ in the basis $\left(a_{1}, \ldots, a_{r}\right)$ we have $M \subset f L$; thus $f$ annihilates $M / L$.

Lemma 1.3 The quotient of a $\mathbb{C}[G]$-module $M$ by a submodule $M^{\prime}$ is torsion if and only $M^{\prime}$ is dense in $M$.

Proof If the quotient is torsion then for any $x \in M$ the intersection of $\mathbb{C}[G] x$ with $M^{\prime}$ is equal to $I x$ for some nonzero ideal $I$; thus it suffices to prove that an ideal is dense in $\mathbb{C}[G]$. This is true: any ideal $I$ contains a nonzero principal ideal $(f)$ (since we are in a Noetherian UFD) and the operator of $\ell^{2}(G)$ induced by multiplication by $f$ is injective, so it has dense image by [17, Lemma 1.14].

If $M^{\prime}$ is dense then its $\ell^{2}$ completion equals that of $M$ and so its rank, which equals its Von Neumann dimension, is the same as that of $M$. Now it is clear that the quotient of a module by a submodule of maximal rank is torsion (see the proof of the above lemma). 
In the same way, given a complex $C_{*}, d_{*}$ of free finitely generated $\mathbb{Z}[G]$-modules we can define its reduced $\ell^{2}$-homology: the $C_{i} \otimes \mathbb{C}$ can be endowed with a $G$-invariant prehilbertian inner product (where we pick a $\mathbb{Z}[G]$-basis and its images under $G$ to be an orthonormal basis) and the differentials $d_{i}$ extend to bounded operators $d_{i}^{(2)}$ from $C_{i}^{(2)}$ to $C_{i-1}^{(2)}$. The reduced $i$ th $\ell^{2}$-homology module $H_{i}^{(2)}(C)$ of $C_{*}$ is then defined to be the $\mathcal{N}(G)$-module $\operatorname{ker}\left(d_{i}^{(2)}\right) / \overline{\operatorname{im}\left(d_{i+1}^{(2)}\right)}$ and the $i$ th $\ell^{2}$-Betti number $b_{i}^{(2)}(C)$ to be its dimension. It follows from the Lemma above that we have $b_{i}^{(2)}(C)=\operatorname{rk}\left(H_{i}(C)\right)$ (see also [17, Lemma 1.34]). The complex $C_{*}$ is thus $\ell^{2}$-acyclic if and only all $H_{i}(C)$ are $\mathbb{Z}[G]$-torsion modules.

\subsection{Alexander polynomials and Fuglede-Kadison determinant}

We now present the analogue for $\mathbb{Z}[G]$-modules of the order of the torsion subgroup for $\mathbb{Z}$-modules, and the Fuglede-Kadison determinant which is linked to the Mahler measure.

Alexander polynomials of $\mathbb{Z}[G]$-modules Let $M$ be a finitely generated $\mathbb{Z}[G]-$ module and $A \in M_{m, n}(\mathbb{Z}[G])$ a presentation matrix for $M$, that is, $M=\mathbb{Z}[G]^{m} / A \mathbb{Z}[G]^{n}$. We define the $l$ th elementary ideal of $M$ as the ideal of $\mathbb{Z}[G]$ generated by the $(m-l)-$ minors of $A$. This definition makes sense since a given presentation matrix for $M$ can be made into any other one by a sequence of elementary transformations that do not affect the elementary ideals (see Turaev [27, Lemma 4.4]). Since $\mathbb{Z}[G]$ is a Noetherian UFD, we can take the greatest common divisor of an ideal; we thus put $\Delta_{l}(M)=\operatorname{gcd}\left(I_{l}\right)$, and call it the $l$ th Alexander polynomial of the module $M$. The Laurent polynomial $\Delta_{l}(M)$ is of course defined only up to multiplication by a unit of $\mathbb{Z}[G]$ and it is zero for all $l \leq \operatorname{rk}(M)$. It depends only on the $\mathbb{Z}[G]$-torsion submodule of $M$; in fact we have (see [27, Chapter 4])

$$
\Delta_{\mathrm{rk}(M)+l}(M)=\Delta_{l}\left(M_{\mathrm{tors}}\right) .
$$

In particular, it follows from this equality that $\Delta_{r}(M)=1$ for a torsion-free module $M$ of rank $r$. We remark that $\Delta_{0}$ is a multiplicative invariant, that is, if we have a short exact sequence $0 \rightarrow M^{\prime} \rightarrow M \rightarrow M^{\prime \prime} \rightarrow 0$ of modules, then $\Delta_{0}(M)=$ $\Delta_{0}\left(M^{\prime}\right) \Delta_{0}\left(M^{\prime \prime}\right)$. To prove this we can suppose the modules are torsion (if not both sides of the equality are zero). Thus $M^{\prime}, M^{\prime \prime}$ have presentation matrices $A^{\prime}, A^{\prime \prime}$ which are of maximal ranks $m^{\prime}, m^{\prime \prime}$; then $M$ has a presentation matrix of the form

$$
A=\left(\begin{array}{cc}
A^{\prime} & * \\
0 & A^{\prime \prime}
\end{array}\right),
$$

whose nonzero $\left(m^{\prime}+m^{\prime \prime}\right)$-minors are products of $m^{\prime}$-minors of $A^{\prime}$ with $m^{\prime \prime}$-minors of $A^{\prime \prime}$. 
Note that the Mahler measure of the Alexander polynomial $\Delta_{l}(M)$ is a well-defined positive real number since the units of $\mathbb{Z}[G]$ are the monomials $\pm t^{v}$ for $v \in \mathbb{Z}^{m}$, whose Mahler measure is equal to one.

Fuglede-Kadison determinant The Fuglede-Kadison determinant is defined for any $\mathcal{N}(G)$-module morphism, see Lück [17, Definition 3.11]; it is an infinite-dimensional analogue of the det' introduced in the next subsection. Here we only need it for those morphisms that are induced from morphisms between free $\mathbb{Z}[G]$-modules. It is always nonzero when the modules are finitely generated. If $A$ is a $\left(n \times n^{\prime}\right)$-matrix with coefficients in $\mathbb{Z}[G]$ we abusively denote by $A$ the continuous operator from $\ell^{2}(G)^{n^{\prime}}$ to $\ell^{2}(G)^{n}$ that it induces, and by $\operatorname{det}_{\mathcal{N}(G)}(A)$ its Fuglede-Kadison determinant. We list some of its properties below for the reader's convenience:

(i) If $A$ is square with nonzero determinant, then we have:

$$
\operatorname{det}_{\mathcal{N}(G)}(A)=\mathcal{M}(\operatorname{det} A) .
$$

(ii) $[17$, Theorem 3.14(1)] If $U \stackrel{f}{\rightarrow} V \stackrel{g}{\rightarrow} W, f$ has dense image and $g$ is injective then

$$
\operatorname{det}_{\mathcal{N}(G)}(g \circ f)=\operatorname{det}_{\mathcal{N}(G)}(f) \operatorname{det}_{\mathcal{N}(G)}(g) .
$$

(iii) [17, Theorem 3.14(2)] If $f_{1}$ has dense image and $f_{2}$ is injective then

$$
\operatorname{det}_{\mathcal{N}(G)}\left(\begin{array}{cc}
f_{1} & f_{3} \\
0 & f_{2}
\end{array}\right)=\operatorname{det}_{\mathcal{N}(G)}\left(f_{1}\right) \operatorname{det}_{\mathcal{N}(G)}\left(f_{2}\right)
$$

(iv) $[17$, Lemma 3.15(3)] We have

$$
\operatorname{det}_{\mathcal{N}(G)}\left(f \mid \begin{array}{l}
\overline{\operatorname{im}(f)} \\
\operatorname{ker}(f)^{\perp}
\end{array}\right)=\operatorname{det}_{\mathcal{N}(G)}(f) .
$$

(v) $\left[17\right.$, Lemma 3.15(4)] Let $f^{*}$ be the adjoint of $f$, then

$$
\operatorname{det}_{\mathcal{N}(G)}\left(f^{*}\right)=\operatorname{det}_{\mathcal{N}(G)}(f)=\sqrt{\operatorname{det}_{\mathcal{N}(G)}\left(f f^{*}\right)} .
$$

We prove (i), which is left as an exercise in [17]. Let $A=L P U$ be a Bruhat decomposition of $A$ over the field $\mathbb{C}(G)$, that is, $P$ is a permutation matrix, $L$ is a lower triangular matrix with diagonal coefficients all equal to 1 and $U$ is an upper triangular matrix with diagonal coefficients $u_{1}, \ldots, u_{n} \in \mathbb{C}(G)$. Let $q$ be the lcm of the denominators of the coefficients of $L$ and $U$. We get $\operatorname{det}_{\mathcal{N}(G)}\left(q^{2} A\right)=\operatorname{det}_{\mathcal{N}(G)}(q L P q U)$; The permutation matrix $P$ is diagonalisable over $\mathbb{C}$ with modulus one eigenvalues, and thus $\operatorname{det}_{\mathcal{N}(G)}(P)=1$. From properties (ii) and (iii) above follow first

$$
\operatorname{det}_{\mathcal{N}(G)}\left(q^{2} I d\right) \operatorname{det}_{\mathcal{N}(G)}(A)=\operatorname{det}_{\mathcal{N}(G)}(q L) \operatorname{det}_{\mathcal{N}(G)}(P) \operatorname{det}_{\mathcal{N}(G)}(q U),
$$


and then

$$
\begin{aligned}
\operatorname{det}_{\mathcal{N}(G)}(A) & =\mathcal{M}(q)^{-2 n} \mathcal{M}\left(q^{n}\right) \mathcal{M}\left(q^{n} u_{1} \ldots u_{n}\right) \\
& =\mathcal{M}\left(u_{1} \ldots u_{n}\right) \\
& =\mathcal{M}(\operatorname{det}(A))
\end{aligned}
$$

\subsection{Reidemeister torsion and $\ell^{2}$-torsion}

Reidemeister torsion Let us first recall some elementary definitions: if $f$ is a map between two finite-dimensional hermitian spaces $V, W$ we $\operatorname{define~}_{\operatorname{det}^{\prime}}(f)=$ $\operatorname{det}\left(\left.f^{*} f\right|_{\operatorname{ker}(f)^{\perp}}\right)$ (the product of all nonzero eigenvalues of the self-adjoint map $f^{*} f$ ). A lattice of $V$ is defined to be a $\mathbb{Z}$-submodule $L$ of $\operatorname{rank} \operatorname{dim}(V)$ spanning $V$ over $\mathbb{C}$; its volume is defined to be the absolute value of the determinant of any map sending an orthonormal basis of $V$ to a $\mathbb{Z}$-basis of $L$. If $V^{\prime}$ is a subspace of $V$ such that $V^{\prime} \cap L$ spans $V^{\prime}$, then this is also true for its orthogonal complement $V^{\prime \prime}$ and we have

$$
\operatorname{vol}(L)=\operatorname{vol}\left(L \cap V^{\prime}\right) \operatorname{vol}\left(L^{\prime \prime}\right)=\operatorname{vol}\left(L^{\prime}\right) \operatorname{vol}\left(L \cap V^{\prime \prime}\right)
$$

where $L^{\prime}, L^{\prime \prime}$ are the orthogonal projections of $L$ on $V^{\prime}, V^{\prime \prime}$. From (1-2) it is easy to deduce the "metric rank formula"

$$
\operatorname{vol}(\operatorname{im}(f))=\frac{\operatorname{det}^{\prime}(f) \operatorname{vol}(L)}{\operatorname{vol}(\operatorname{ker} f)} .
$$

Now let $C_{*}, d_{*}$ be a finite complex of free $\mathbb{Z}[G]$-modules; we pick for each $i$ a $\mathbb{Z}$-basis of $C_{i}$ and endow $C_{*} \otimes \mathbb{C}$ with the inner product where all these bases are orthonormal. The free part $H_{i}(C)_{\text {free }}$ of the homology is a lattice in $H_{i}(C \otimes \mathbb{C})$, which itself can be seen as the orthogonal of $\operatorname{im}\left(d_{i+1}\right)$ in $\operatorname{ker}\left(d_{i}\right) \otimes \mathbb{C}$. Then the Reidemeister torsion defined by

$$
\tau\left(C_{*}\right)=\prod_{i} \operatorname{det}^{\prime}\left(d_{i}\right)^{(-1)^{i}}
$$

does not depend on the choice of the bases and is in fact given by the equality

$$
\tau\left(C_{*}\right)=\prod_{i}\left({\frac{\left|H_{i}(C)_{\mathrm{tors}}\right|}{\operatorname{vol}\left(H_{i}(C)_{\mathrm{free}}\right)}}^{(-1)^{i+1}}\right) .
$$

The proof of this equality is a simple exercise using (1-3). 
$\ell^{2}$-torsion Let $C_{*}, d_{*}$ be a finite complex of free $\mathbb{Z}[G]$-modules. By analogy with the finite-dimensional case we put

$$
\tau^{(2)}(C)=\prod_{i} \operatorname{det}_{\mathcal{N}(G)}\left(d_{i}\right)^{(-1)^{i}}
$$

In our particular case this can be computed:

Proposition 1.4 In the situation above, suppose that $C_{*}$ is $\ell^{2}$-acyclic. Then we have

$$
\tau^{(2)}(C)=\prod_{i} \mathcal{M}\left(\Delta_{0}\left(H_{i}(C)\right)\right)
$$

Proof This can be deduced without too much effort from the equality in Turaev [27, Theorem 4.7]. We do not do so here, and instead prove a result which is also valid for non $\ell^{2}$-acyclic complexes (Lemma B.3), following the lines of the proof of (1-4). The equality (1-5) follows immediately from this result.

Example: link complements Let $X$ be a CW-structure on the complement of a regular neighbourhood $L$ of a link in the three-sphere, and put $G=H_{1}(X)$. Let $\widehat{X}$ be the maximal abelian cover of $X$; then $\widehat{X}$ is a $G-\mathrm{CW}$-complex. The only $H_{i}(\widehat{X})$ that can contain $\mathbb{Z}[G]$-torsion is $H_{1}$, and it is known that in the case of a knot $K, H_{1}$ is in fact a torsion module. In this case we get from Proposition 1.4 that

$$
\tau^{(2)}(\widehat{X})=\mathcal{M}(\Delta(K))
$$

where $\Delta(K)$ is the Alexander polynomial of $K$. For a link with nonzero first Alexander polynomial $\Delta(L)$ we get in the same way that $\tau^{(2)}(\widehat{X})=\mathcal{M}(\Delta(K))$; if $\Delta(L)=0$ then the $\ell^{2}$-torsion depends on the $\mathrm{CW}$-structure; the precise dependance is computed in Lemma B.3.

\section{Approximation for the Fuglede-Kadison determinant}

Let $H \subset G$ be a subgroup and $M$ a $\mathbb{Z}[G]$-module. We denote by $M_{H}$ the $\mathbb{Z}[G / H]$ module obtained by restricting from $G$ to $G / H$ :

$$
M_{H}=\mathbb{Z}[G / H] \otimes_{\mathbb{Z}[G]} M
$$

There is a $G / H$-equivariant isomorphism from $M_{H}$ to the quotient module $M /(H-$ 1) $M$. If $f: \mathbb{Z}[G]^{n} \rightarrow \mathbb{Z}[G]^{n^{\prime}}$ is a linear map of free modules, $f_{H}$ is the map from $\mathbb{Z}[G / H]^{n} \rightarrow \mathbb{Z}[G / H]^{n^{\prime}}$ that it induces. The restricted determinant $\operatorname{det}^{\prime}\left(f_{H}\right)$ is always 
taken with respect to the canonical metric on $\mathbb{C}[G / H]$, for which the elements of $G / H$ are an orthonormal basis. Let $C_{*}, d_{*}$ be a complex of $\mathbb{Z}[G]$-modules; then $C_{H}$ is the $\mathbb{Z}[G / H]$-complex with $C_{*, H}=\left(C_{*}\right)_{H}$ and $d_{*, H}=\left(d_{*}\right)_{H}$.

It is a well-known result of Lück (see [16] or [17, Chapter 13]) that for any complex $C_{*}$ of free $\mathbb{Z}[G]$-modules the normalised Betti numbers converge to the $\ell^{2}$ Betti number, that is,

$$
\lim _{\alpha(H) \rightarrow \infty} \frac{\operatorname{dim}\left(H_{i}\left(C_{H}\right) \otimes \mathbb{C}\right)}{[G: H]}=b_{i}^{(2)}\left(C_{*}\right) .
$$

This result is true for any residually finite group and any exhaustive sequence of finite index normal subgroups. It is a natural question to ask whether a similar result holds for torsions, that is, does the sequence $\operatorname{det}^{\prime}\left(f_{H}\right)^{\frac{1}{[G: H]}}$ converge to $\operatorname{det}_{\mathcal{N}(G)}(f)$. We shall prove the following result.

Proposition 2.1 Let $A \in M_{n, n^{\prime}}(\mathbb{Z}[G])$ be any matrix. Then

$$
\limsup _{\alpha(H) \rightarrow \infty} \frac{\log \left(\operatorname{det}\left(A_{H}\right)\right)}{[G: H]}=\log \left(\operatorname{det}_{\mathcal{N}(G)}(A)\right) .
$$

More precisely, the result we prove and use in the next section is:

Proposition 2.2 Let $A_{1}, \ldots, A_{n}$ be a finite collection of matrices with coefficients in $\mathbb{Z}[G]$; there exists a sequence $G_{N}$ of subgroups of finite index in $G$ such that $\alpha\left(G_{N}\right) \underset{N \rightarrow \infty}{\longrightarrow} \infty$ and for all $i$ we have:

(i) $\lim _{N \rightarrow \infty} \frac{\log \operatorname{det}^{\prime}\left(A_{i, G_{N}}\right)}{\left[G: G_{N}\right]}=\log \operatorname{det}_{\mathcal{N}(G)}\left(A_{i}\right)$,

(ii) $\left|\operatorname{dim}\left(\operatorname{ker}\left(A_{i, G_{N}}\right) \otimes \mathbb{C}\right)-\left[G: G_{N}\right] \operatorname{rk}\left(\operatorname{ker}\left(A_{i}\right)\right)\right|=O\left(\log \left[G: G_{N}\right]\right)$.

Proposition 2.1 follows from this since arguing as in the proof of Lemma 2.6 below it is easy to see that

$$
\limsup _{\alpha(H) \rightarrow \infty}\left(\operatorname{det}^{\prime}\left(A_{H}\right)^{\frac{1}{[G: H]}}\right) \leq \operatorname{det}_{\mathcal{N}(G)}(A)
$$

(this is a general fact, see Lück [16, Theorem 3.4(2)]). 


\subsection{Growth of Betti numbers}

We shall need precise estimates on the speed of convergence in (2-1). For a subgroup $H \subset \mathbb{Z}^{m}$ we set

$$
H^{\perp}=\left\{\left(\zeta_{1}, \ldots, \zeta_{m}\right) \in \mathbb{T}^{m}, \forall\left(v_{1}, \ldots, v_{m}\right) \in H, \zeta_{1}^{v_{1}} \ldots \zeta_{m}^{v_{m}}=1\right\} .
$$

The result we want is the following (we count the multiplicities when computing the number of zeroes of some polynomial):

Proposition 2.3 We remind the reader that $G=\mathbb{Z}^{m}$ and that we identify the group ring $\mathbb{Z}[G]$ with the ring of Laurent polynomials $\mathbb{Z}\left[t_{1}^{ \pm 1}, \ldots ; t_{m}^{ \pm 1}\right]$. Let $M$ be a finitely generated $\mathbb{Z}[G]-$ module (resp. $C_{*}$ a finite complex of finitely generated free $\mathbb{Z}[G]-$ modules). Then there exists a polynomial $P \in \mathbb{Z}\left[t_{1}, \ldots, t_{m}\right]$ such that for all subgroups $H \subset G$ we have

$$
\begin{aligned}
& \left|\operatorname{dim}\left(M_{H} \otimes \mathbb{C}\right)-[G: H] \operatorname{rk}(M)\right| & \leq \mid\left\{\zeta \in H^{\perp}, P(\zeta)\right. & =0\} \mid \\
\text { (resp. } & \left|b_{i}\left(C_{H}\right)-[G: H] b_{i}^{(2)}\left(C_{*}\right)\right| & \leq \mid\left\{\zeta \in H^{\perp}, P(\zeta)\right. & =0\} \mid) .
\end{aligned}
$$

Proof Let $M$ be a finitely generated $\mathbb{Z}[G]$-module; let $i: L \hookrightarrow M$ be an embedding of a free module of maximal rank in $M$ and $T$ the torsion module $M / L$. We see that

$$
\left|\operatorname{dim}\left(M_{H} \otimes \mathbb{C}\right)-[G: H] \operatorname{rk}(M)\right| \leq \operatorname{dim}\left(T_{H} \otimes \mathbb{C}\right)+\operatorname{dim}\left(\operatorname{ker}\left(i_{H}\right) \otimes \mathbb{C}\right) .
$$

We need to bound the right-hand side; this is done in the two next lemmas.

Lemma 2.4 If $T$ is a finitely generated torsion $\mathbb{Z}[G]$-module there is a polynomial $P$ such that we have

$$
\operatorname{dim}\left(T_{H} \otimes \mathbb{C}\right) \leq\left|\left\{\zeta \in H^{\perp}, P(\zeta)=0\right\}\right| .
$$

Proof Let $a_{1}, \ldots, a_{s}$ be generators for $T$, since they are torsion elements there exists a nonzero $P \in \mathbb{Z}\left[t_{1}, \ldots, t_{m}\right]$ such that $P a_{i}=0$ for all $i$. Then there is a surjection from $(\mathbb{C}[G] /(P))^{k}$ to $T$, and we have

$$
\operatorname{dim}\left(T_{H} \otimes \mathbb{C}\right) \leq k \operatorname{dim}(\mathbb{C}[G] /(P) \otimes \mathbb{C}[G / H]) .
$$

The dimension on the right is equal to $\left|H^{\perp} \cap\{P=0\}\right|$ : we have a surjection $\mathbb{C}[G / H] \rightarrow$ $(\mathbb{C}[G] /(P))_{H}$, and in the decomposition $\mathbb{C}[G / H]=\sum_{\zeta \in H^{\perp}} \mathbb{C}_{\zeta}$ (where $\mathbb{C}_{\zeta}$ is a complex line on which $G / H$ acts by the character associated with $\zeta$ ) the kernel of this 
surjection is $\bigoplus_{\zeta, P(\zeta) \neq 0} \mathbb{C}_{\zeta}$. Thus $\operatorname{dim}\left(T_{H}\right)$ is bounded by the number of zeroes of $P^{k}$ (recall that we count multiplicities).

Lemma 2.5 Let $i: M_{1} \rightarrow M_{2}$ be an injective map of $\mathbb{Z}[G]$-modules. Then there is a polynomial $Q$ such that for all $H$ we have

$$
\operatorname{dim}\left(\operatorname{ker}\left(i_{H}\right) \otimes \mathbb{C}\right) \leq\left|\left\{\zeta \in H^{\perp}, Q(\zeta)=0\right\}\right| .
$$

Proof We first consider the case where $M \subset \mathbb{Z}[G]^{n}$ is a submodule and $i$ its embedding. We begin by proving there is a $Q_{1}$ such that

$$
\operatorname{dim}\left(M_{H} \otimes \mathbb{C}\right) \leq[G: H] \operatorname{rk}(M)+\left|\left\{\zeta \in H^{\perp}, Q_{1}(\zeta)=0\right\}\right| .
$$

To prove this take a torsion module $T=M / L$ where $L \subset M$ is a free maximal rank submodule: the image of $L_{H} \otimes \mathbb{C}$ in $M_{H} \otimes \mathbb{C}$ has dimension less than $\operatorname{dim}\left(L_{H} \otimes \mathbb{C}\right)=$ $\operatorname{rk}(M)[G: H]$ and codimension equal to $\operatorname{dim}\left(T_{H} \otimes \mathbb{C}\right)$ so that we can take for $Q_{1}$ the polynomial associated to $T$ by Lemma 2.4 .

Now let $M^{\prime} \subset \mathbb{Z}[G]^{n}$ be a submodule such that $M \cap M^{\prime}=0$ and $\operatorname{rk}(M)+\operatorname{rk}\left(M^{\prime}\right)=n$ and $i^{\prime}$ its embedding. The quotient $T^{\prime}:=\mathbb{Z}[G]^{n} / M \oplus M^{\prime}$ is a torsion module and so from Lemma 2.4 we get a polynomial $Q_{2}$ such that

$$
\operatorname{dim}\left(T_{H}^{\prime} \otimes \mathbb{C}\right) \leq\left|\left\{\zeta \in H^{\perp}, Q_{2}(\zeta)=0\right\}\right| .
$$

On the other hand we have an exact sequence

$$
0 \rightarrow M \oplus M^{\prime} \rightarrow \mathbb{Z}[G]^{n} \rightarrow T^{\prime} \rightarrow 0
$$

which becomes after tensorization with $\mathbb{Z}[G / H]$

$$
M_{H} \oplus M_{H}^{\prime} \rightarrow \mathbb{Z}[G / H]^{n} \rightarrow T_{H}^{\prime} \rightarrow 0 .
$$

We get

$\operatorname{dim}\left(\operatorname{ker}\left(i_{H}\right) \otimes \mathbb{C}\right)+\operatorname{dim}\left(\operatorname{ker}\left(i_{H}^{\prime}\right) \otimes \mathbb{C}\right)=\operatorname{dim}\left(T_{H}^{\prime} \otimes \mathbb{C}\right)+\operatorname{dim}\left(M_{H} \oplus M_{H}^{\prime} \otimes \mathbb{C}\right)-n[G: H]$.

We can apply the reasoning leading to (2-2) to $M^{\prime}$ and this yields a polynomial $Q_{3}$ such that $\operatorname{dim}\left(M_{H}^{\prime} \otimes \mathbb{C}\right)-[G: H] \operatorname{rk}\left(M^{\prime}\right)$ is less than the number of zeroes of $Q_{3}$ on $H^{\perp}$; letting $Q=Q_{1} Q_{2} Q_{3}$, (2-2) and (2-3) above and this imply that the right hand side is bounded above by the number of zeroes of $Q$ in $H^{\perp}$.

In the general case we have an embedding of $M_{1} /\left(M_{1}\right)_{\text {tors }}$ in a free module $\mathbb{Z}[G]^{n}$ : let $i^{\prime}$ be the embedding of $M_{2} /\left(M_{2}\right)_{\text {tors }}$ in $\mathbb{Z}[G]^{n}$ this yields. We get Lemma 2.5 by applying the preceding discussion to $i^{\prime}$ and Lemma 2.4 to $\left(M_{2}\right)_{\text {tors }}$. 
Let $C_{*}$ be a finite complex of free $\mathbb{Z}[G]$-modules; we use standard arguments to deduce the second point of the proposition from the first one. Let $n_{i}=\operatorname{rk}_{\mathbb{Z}[G]}\left(C_{i}\right)$; then we have $\operatorname{rk}_{\mathbb{Z}}\left(C_{i, H}\right)=[G: H] n_{i}$, and it follows that

$$
\begin{aligned}
b_{i}\left(C_{H}\right) & =\operatorname{rk}_{\mathbb{Z}}\left(\operatorname{ker}\left(d_{i, H}\right)\right)-\operatorname{rk}_{\mathbb{Z}}\left(\operatorname{im}\left(d_{i+1, H}\right)\right) \\
& =\operatorname{rk}_{\mathbb{Z}}\left(\operatorname{ker}\left(d_{i, H}\right)\right)+n_{i+1}[G: H]-\operatorname{rk}_{\mathbb{Z}}\left(\operatorname{ker}\left(d_{i+1, H}\right)\right) .
\end{aligned}
$$

On the other hand,

$$
b_{i}^{(2)}(C)=\mathrm{rk}_{\mathbb{Z}[G]}\left(\operatorname{ker}\left(d_{i}\right)\right)+n_{i+1}-\mathrm{rk}_{\mathbb{Z}[G]}\left(\operatorname{ker}\left(d_{i+1}\right)\right)
$$

so that we need only prove that for a matrix $A \in M_{n, n^{\prime}}(\mathbb{Z}[G])$ the difference

$$
\mathrm{rk}_{\mathbb{Z}}\left(\operatorname{ker}\left(A_{H}\right)\right)-[G: H] \mathrm{rk}_{\mathbb{Z}[G]}(\operatorname{ker}(A))
$$

is bounded by the number of zeroes in $H^{\perp}$ of some polynomial. We know from the case of a module and Lemma 2.5 that

$$
\operatorname{rk}_{\mathbb{Z}} \operatorname{im}\left(A_{H}\right)=[G: H] \mathrm{rk}_{\mathbb{Z}[G]}(\operatorname{im} A)+e
$$

where $e$ is the number of zeroes in $H^{\perp}$ of some polynomial, and since $\operatorname{rk}_{\mathbb{Z}} \operatorname{im}\left(A_{H}\right)+\operatorname{rk}_{\mathbb{Z}} \operatorname{ker}\left(A_{H}\right)=n^{\prime}[G: H]=\left(\operatorname{rk}_{\mathbb{Z}[G]} \operatorname{im}(A)+\operatorname{rk}_{\mathbb{Z}[G]} \operatorname{ker}(A)\right)[G: H]$ we get the result we want.

There is a majoration of the number of zeroes of a polynomial on the $m$-torus: given an affine subvariety $X \subset \mathbb{C}^{m}$ we denote by $X[H]$ the finite set $H^{\perp} \cap X$; then there exists a constant $C$ depending only on $X$ such that

$$
|X[H]| \leq C \frac{[G: H]}{\alpha(H)} .
$$

We can retrieve from Proposition 2.3 and this inequality a generalisation to the non $\ell^{2}$-acyclic case of Clair-Whyte [3, Theorem 2.1] (where a proof of the above bound can be found).

Corollary Let $M$ be a finitely generated $\mathbb{Z}[G]$-module (resp. $C_{*}$ a finite complex of finitely generated free $\mathbb{Z}[G]$-modules). Then there exists a constant $C>0$ such that

$$
\begin{aligned}
\left|\operatorname{dim}\left(M_{H} \otimes \mathbb{Q}\right)-[G: H] \operatorname{rk}(M)\right| & \leq C \frac{[G: H]}{\alpha(H)} \\
\left|b_{i}\left(C_{H}\right)-[G: H] b_{i}^{(2)}\left(C_{*}\right)\right| & \left.\leq C \frac{[G: H]}{\alpha(H)}\right) .
\end{aligned}
$$

(resp. 


\subsection{Convergence of Riemann sums}

We shall soon give a method to construct from a finite number of polynomials in $m$ variables a sequence of subgroups $G_{N}$ of $G$ for which the Riemann sums over the subsets $G_{N}^{\perp}$ converge to the Mahler measure, and the polynomials have "few" zeroes in $G_{N}^{\perp}$. We will also need a lower bound on the nonzero values of the polynomials at points in $G_{N}^{\perp}$. In the case where $G$ is cyclic these are two well-known facts which we recall below.

Lemma 2.6 Let $P \in \mathbb{Z}[t]$ be a nonzero polynomial;

(i) $\lim _{N \rightarrow \infty} \frac{1}{N} \sum_{\substack{\xi^{N}=1 \\ P(\zeta) \neq 0}} \log |P(\zeta)|=m(P)$.

(ii) There is a $C_{P}>0$ such that for all $N$ th roots of unity $\zeta$ such that $P(\zeta) \neq 0$ we have: $\log |P(\zeta)| \geq-C_{P} \log N$.

Proof From the additivity of the logarithmic Mahler measure and the decomposition of $P$ into irreducible factors over $\mathbb{C}$ we see that the only case we need consider is that of a degree one polynomial of the form $t-a$ for some algebraic number $a$; if the absolute value of $a$ is not 1 then the result is trivial. If $|a|=1$ then it is easy to see that the superior limit of the Riemann sums is less than 0 (just cut off $\log |z-a|$ near $a$ to replace it with a continuous function with arbitrarily small mean value over $\mathbb{T}^{1}$ ). Jensen's formula implies that the Mahler measure of $z-a$ is 0 , so we have to prove that

$$
\liminf _{N \rightarrow \infty} \frac{1}{N} \sum_{\substack{\zeta^{N}=1 \\ \zeta \neq a}} \log |\zeta-a| \geq 0 .
$$

From a diophantine estimate due to Baker it is deduced in Everest-Ward [4, Lemma 1.11] that there exists a constant $C_{a}>0$ such that

$$
\prod_{\substack{\zeta^{N}=1 \\ \zeta \neq a}}|\zeta-a| \geq \frac{1}{N^{C_{a}}} .
$$

we get for all $N$

$$
\frac{1}{N} \sum_{\substack{\zeta^{N}=1 \\ \zeta \neq a}} \log |\zeta-a| \geq \frac{-C_{a} \log N}{N}
$$

and since the right-hand side converges to zero we get the desired result.

Statement (ii) is a "local" version of (2-5) and is easily deduced from it. 
If $m>1$ the same argument as above shows that

$$
\limsup _{\alpha(H) \rightarrow \infty} \sum_{\substack{\zeta \in H^{\perp} \\ P(\zeta) \neq 0}} \log |P(\zeta)| \leq m(P) .
$$

However, we cannot give good lower bounds for the values of $P$ at roots of unity and thus cannot prove the convergence. As a palliative, we use in the next subsection an approximation result due to Boyd and Lawton to be able to apply the above results to multivariate polynomials.

\subsection{Construction of the sequence $G_{N}$}

Proposition 2.7 Let $\mathcal{P}$ be a finite collection of polynomials in $\mathbb{Z}\left[t_{1}, \ldots, t_{m}\right]$. There exists a sequence of finite index subgroups $G_{N} \subset G=\mathbb{Z}^{m}$ such that $\alpha\left(G_{N}\right) \underset{N \rightarrow \infty}{\longrightarrow} \infty$ and for any polynomial $P \in \mathcal{P}$ we have

(i) $\frac{1}{\left[G: G_{N}\right]} \sum_{\substack{\zeta \in G_{N}^{\perp} \\ P(\zeta) \neq 0}} \log |P(\zeta)| \underset{N \rightarrow \infty}{\longrightarrow} m(P) ;$

(ii) $\left|\left\{\zeta \in G_{N}^{\perp}, P(\zeta)=0\right\}\right| \leq \log \left[G: G_{N}\right]$;

(iii) for all $\zeta \in G_{N}^{\perp}, \log |P(\zeta)| \geq-\left(\log \left[G: G_{N}\right]\right)^{2}$.

Proof It is clear from the nature of the arguments below that we need only consider the case of a single polynomial $P \in \mathbb{Z}\left[t_{1}, \ldots, t_{m}\right]$.

For any vector $v=\left(v_{1}, \ldots, v_{m}\right) \in \mathbb{Z}^{m}$ we denote by $v^{*}$ the subgroup given by $\left\{\left(u_{1}, \ldots, u_{m}\right) \in \mathbb{Z}^{m}, \sum_{i} u_{i} v_{i}=0\right\}$. We put $q(v)=\alpha\left(v^{*}\right)$ and for a polynomial $P \in \mathbb{Z}\left[t_{1}, \ldots, t_{m}\right], P_{v}(X)=P\left(X^{v_{1}}, \ldots, X^{v_{m}}\right)$. The main result of Lawton [12] is that

$$
m\left(P_{v}\right) \underset{q(v) \rightarrow \infty}{\longrightarrow} m(P) .
$$

For a prime $p$ we denote by $p=p_{1}<p_{2}<\cdots<p_{m}$ the $m$ primes following $p$; we put $r_{i}=\prod_{j \neq i} p_{j}$ for $i=1, \ldots, m$ and $r=\left(r_{1} ; \ldots, r_{m}\right)$. It is easy to see that $q(r) \geq p$ : if $\sum_{i} s_{i} r_{i}=0$ for some nonzero $s \in \mathbb{Z}^{m}$ and if $s_{j} \neq 0$ we get $-t s_{j} r_{j}=p_{j} \sum_{i \neq j} \frac{r_{i}}{p_{j}} s_{j}$. Since the prime $p_{j}$ does not divide $r_{j}$ we must have $p_{j} \mid s_{j}$ and thus $q(r) \geq\left|s_{j}\right| \geq p_{j} \geq p$. Thus $m\left(P_{r}\right) \underset{p \rightarrow \infty}{\longrightarrow} m(P)$. For an integer $M \geq 0$ we define a subgroup $G_{p, M}$ of $G$ by

$$
G_{p, M}=\left\{v \in G, \sum_{i} v_{i} r_{i} \equiv 0(\bmod M)\right\} ;
$$

this is equal to $r^{*} \oplus \mathbb{Z} M v$ for any $v$ generating a supplement to $r^{*}$, and has index $M$ in $G$. It is not hard to see that for $M>m p_{1} \ldots p_{m}$ we have $\alpha\left(G_{p, m}\right) \geq p$ : let 
$v \in G_{p, M}, v \notin r^{*}$. Then $\left|\sum_{i} r_{i} v_{i}\right| \geq M$ and so we must have $\left|v_{j}\right|>M /\left(m \prod_{i \neq j} p_{i}\right)$ for some $j$. This is bigger than $p$ for $M$ as above and the claim follows. Now given an $\varepsilon>0$, choose $p$ big enough so that $\left|m\left(P_{r}\right)-m(P)\right|<\varepsilon$; by Lemma 2.6 there exists an integer $M_{0}$ such that for all $M \geq M_{0}$,

$$
\left|m\left(P_{r}\right)-\frac{1}{M} \sum_{\zeta^{M}=1, P_{r}(\zeta) \neq 0} \log \right| P_{r}(\zeta)||<\varepsilon .
$$

We see that $G_{p, M}^{\perp}=\left\{\left(\zeta^{r_{1}}, \ldots, \zeta^{r_{m}}\right), \zeta^{M}=1\right\}$ and thus we get

$$
\left|m(P)-\frac{1}{\left[G: G_{p, M}\right]} \sum_{\zeta \in G_{p, M}^{\perp}, P(\zeta) \neq 0} \log \right| P(\zeta)||<2 \varepsilon
$$

for any $M \geq M_{0}$.

Now the number of zeroes of $P$ in $G_{p, M}^{\perp}$ is less than the degree of $P_{r}$, which is itself less than

$$
\max _{i}\left|r_{i}\right| \times \operatorname{deg}(P) \leq \operatorname{deg}(P) p_{1} \ldots p_{m} \leq \operatorname{deg}(P) 2^{m(m+1) / 2} p^{m}
$$

since we have $p_{i} \leq 2 p_{i-1}$ by a well-known result of Chebyshev. Thus there exists an integer $M_{1}$ such that the number of zeroes of $P$ in $G_{p, M}$ is less than $\log (M)$ for all $M \geq M_{1}$.

From Lemma 2.6 we get that for all $p$ there is a constant $C_{p}$ such that the nonzero values of $P$ at the points $\zeta \in G_{p, M}$ satisfy

$$
\log |P(\zeta)| \geq-C_{p} \log (M) .
$$

It follows that for $M \geq M_{2}=\exp \left(C_{p}\right)$ we have $\log |P(\zeta)| \geq-\log (M)^{2}$.

We can construct a sequence $G_{N}=G_{p_{N}, M_{N}}$ where $p_{N}$ is the $N$ th prime number and $M_{N}$ a well-chosen positive integer: we take $M_{N}>m p_{N} \ldots p_{N+m-1}$ so that $\alpha\left(G_{p_{N}, M_{N}}\right) \geq p_{N}, M_{N} \geq M_{0}, M_{1}, M_{2}\left(M_{0}\right.$ as above for, say, $\left.\varepsilon=1 / p\right)$ so that (i), (ii) and (iii) hold.

\subsection{Proof of Proposition 2.2}

We shall need the following elementary lemma:

Lemma 2.8 Let $f$ be a linear map between Hermitian spaces $V, W$ and $V^{\prime}$ a subspace in $V$ with codimension $d$; suppose that any singular value $\lambda$ of $f$ satisfies $c \leq \lambda \leq C$. Putting $f^{\prime}=f_{\mid V^{\prime}}$, we have

$$
d \log (c) \leq \log \left(\operatorname{det}^{\prime}(f)\right)-\log \left(\operatorname{det}^{\prime}\left(f^{\prime}\right)\right) \leq d \log (C) .
$$


Proof If $\lambda_{1} \geq \ldots \geq \lambda_{\operatorname{dim} V}$ are the eigenvalues of an invertible positive self-adjoint endomorphism $g$ of $V$, we have

$$
\lambda_{i}=\max _{F \leq V, \operatorname{dim}(F)=i} \min _{x \in F} \frac{\|g x\|}{\|x\|}
$$

so if $g=\left.f^{*} f\right|_{\operatorname{ker}(f)^{\perp}}$ and $\lambda_{1}^{\prime}, \ldots, \lambda_{\operatorname{dim} V-d}^{\prime}$ are its eigenvalues in restriction to $V^{\prime}$ (the singular values of $f^{\prime}$ ), we have $\lambda_{i} \geq \lambda_{i}^{\prime} \geq \lambda_{i+d}$ for all $i \geq 0$ and it follows that

$$
\log \left(\operatorname{det}^{\prime}(f)\right)-\log \left(\operatorname{det}^{\prime}\left(f^{\prime}\right)\right) \leq \sum_{i=1}^{d} \log \left(\lambda_{i}\right) \leq d \log (C)
$$

and that $\log \left(\operatorname{det}^{\prime}(f)\right)-\log \left(\operatorname{det}^{\prime}\left(f^{\prime}\right)\right) \geq \sum_{i=\operatorname{dim} V-d}^{\operatorname{dim} V} \log \left(\lambda_{i}\right) \geq d \log (c)$.

This completes the proof.

Proof of Proposition 2.2 As in the proof of Proposition 2.7 it is clear that we can restrict ourselves to the case of a single matrix. The second thing to be acknowledged is that it suffices to prove the result for self-adjoint matrices since for any matrix $A \in M_{n, m}(\mathbb{C}[G])$ we have $\operatorname{det}_{\mathcal{N}(G)}(A)=\sqrt{\operatorname{det}_{\mathcal{N}(G)}\left(A^{*} A\right)}$. Thus we suppose that $A \in M_{n}(\mathbb{Z}[G])$ is a self-adjoint matrix.

We want to show that

$$
\lim _{N \rightarrow \infty} \frac{\log \operatorname{det}^{\prime}\left(A_{G_{N}}\right)}{\left[G: G_{N}\right]}=\operatorname{det}_{\mathcal{N}(G)}(A)
$$

for a sequence $G_{N}$ obtained from Proposition 2.7. The strategy is to use Lemma 2.8 above to reduce to the case when $A$ has a nonzero determinant. The proposition is then a straightforward consequence of (i) of Proposition 2.7 applied to $\operatorname{det}(A)$. Let $L$ be a free maximal rank $\mathbb{Z}[G]$-submodule in $\operatorname{im}(A), L^{\prime}$ its image by $A$ and $h, g$ be isomorphisms of $\mathbb{Z}[G]^{r}(r=\operatorname{rk}(A))$ with $L, L^{\prime}$ so that the following diagram commutes

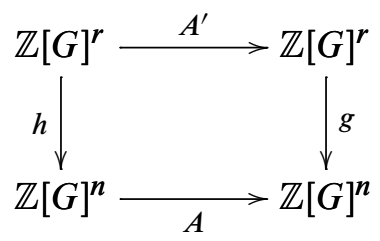

with $A^{\prime}$ a square matrix with nonzero determinant. Since $L \otimes \mathbb{C}$ is dense in $\operatorname{ker}(A)^{\perp}$, Lück [17, Lemma 3.15(3)] yields the equality

$$
\operatorname{det}_{\mathcal{N}(G)}(A)=\operatorname{det}_{\mathcal{N}(G)}(g) \operatorname{det}_{\mathcal{N}(G)}\left(A^{\prime}\right) \operatorname{det}_{\mathcal{N}(G)}\left(h^{-1}\right) .
$$


We write the characteristic polynomial $\operatorname{det}(t-A)$ as $\sum_{i=i_{0}}^{n} P_{i}\left(t_{1}, \ldots, t_{m}\right) t^{i}$ where $i_{0}$ is the rank of $\operatorname{ker}(A)$ and thus all $P_{i}$ are nonzero. Let $\mathcal{P}$ be the collection of all $P_{i}$ together with the nonzero coefficients of the characteristic polynomials of $A^{\prime}, h h^{*}$ and $g g^{*}$ and all the polynomials appearing in Lemma 2.5 for $h$ and $g$ and the polynomials from Lemma 2.4 for $\operatorname{im}(A) / \operatorname{im}(h)$ and $\operatorname{im}(A) / \operatorname{im}(g)$. Let $G_{N}$ be the sequence associated to $\mathcal{P}$ by Proposition 2.7. Since $\operatorname{det}^{\prime} A(\zeta)=P_{i}(\zeta)$ for $i=\operatorname{dim} \operatorname{ker}(A(\zeta)) \geq i_{0}$ (and similarly for the other matrices) we get by (iii) of Proposition 2.7 that for any singular value $\lambda$ of $A_{G_{N}}, A_{G_{N}}^{\prime}, h_{G_{N}}$ or $g_{G_{N}}$ we have

$$
\log \lambda \geq-\log \left[G: G_{N}\right]^{2} .
$$

From Lemma 2.5 and (ii) of Proposition 2.7 we see that the subspace $\operatorname{ker}\left(h_{G_{N}}\right)+$ $\operatorname{ker}\left(g_{G_{N}}\right)$ of $\mathbb{C}\left[G / G_{N}\right]^{r}$ has dimension $\leq \log \left[G: G_{N}\right]$. In the same way Lemma 2.4 yields that $\operatorname{im}\left(h_{G_{N}}\right)$ and $\operatorname{im}\left(g_{G_{N}}\right)=A_{H} \operatorname{im} h_{G_{N}}$ have codimension $\leq \log \left[G: G_{N}\right]$ in $\operatorname{ker}\left(A_{H}\right)^{\perp}$. We define subspaces $V_{1} \subset \mathbb{C}\left[G / G_{N}\right]^{r}$ and $V_{2} \subset \mathbb{C}\left[G / G_{N}\right]^{n}$ by

$$
\begin{aligned}
& V_{1}=\operatorname{ker}\left(h_{G_{N}}\right)^{\perp} \cap \operatorname{ker}\left(A_{G_{N}}^{\prime}\right) \cap A_{G_{N}}^{\prime-1}\left(\operatorname{ker}\left(g_{G_{N}}\right)^{\perp}\right) \\
& V_{2}=h_{G_{N}}\left(V_{1}\right)
\end{aligned}
$$

so that we have a commutative diagram

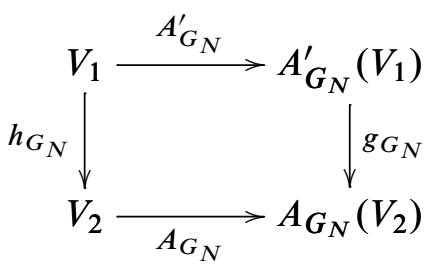

such that all arrows are isomorphisms. We also get, for $i=1,2$,

$$
\operatorname{dim} V_{i}-r\left[G: G_{N}\right]=O\left(\log \left[G: G_{N}\right]\right) .
$$

In the sequel we use the abbreviation $u_{H} \sim v_{H}$ to mean that

$$
\log \left(u_{H}\right)=\log \left(v_{H}\right)+o([G: H]) \text {. }
$$

From Lemma 2.8 and (2-6) above we see that for any linear map $f: \mathbb{Z}[G]^{n} \rightarrow \mathbb{Z}[G]^{n^{\prime}}$ and any subspaces $F_{N} \subset \mathbb{C}\left[G / G_{N}\right]^{n}$ such that $\operatorname{dim}\left(F_{N}\right)=O\left(\left[G: G_{N}\right]^{b}\right)$ for some $b<1$ we have $\operatorname{det}^{\prime}\left(f_{G_{N}}\right) \sim \operatorname{det}^{\prime}\left(\left.f_{G_{N}}\right|_{F_{N}}\right)$. Applying this to $f=A^{\prime}$, with Lemma 2.8 applied to $V=W=\mathbb{C}\left[G / G_{N}\right]^{r}$ and $V^{\prime}=V_{1}$ we get

$$
\operatorname{det}^{\prime}\left(A_{G_{N}}^{\prime}\right) \sim \operatorname{det}^{\prime}\left(A_{G_{N}}^{\prime} \mid V_{1}\right) .
$$


In the same way we obtain

$$
\begin{aligned}
\operatorname{det}^{\prime}\left(A_{G_{N}}^{\prime} \mid V_{1}\right) & =\operatorname{det}^{\prime}\left(\left(\left.g_{G_{N}}\right|_{A_{G_{N}} V_{2}} ^{A_{G_{N}} V_{1}}\right)^{-1} A_{G_{N}} h_{G_{N}} \mid V_{1}\right) \\
& =\operatorname{det}\left(g_{G_{N}} \mid \begin{array}{l}
A_{G_{N}} V_{2} \\
A_{G_{N}}^{\prime} V_{1}
\end{array}\right)^{-1} \operatorname{det}\left(A_{G_{N}} \mid V_{2}\right) \operatorname{det}\left(h_{G_{N}} \mid V_{1}\right) \\
& \sim \operatorname{det}^{\prime}\left(g_{G_{N}}\right)^{-1} \operatorname{det}^{\prime}\left(A_{G_{N}}\right) \operatorname{det}^{\prime}\left(h_{G_{N}}\right)
\end{aligned}
$$

where the last line follows from Lemma 2.8 applied to $f=g_{G_{N}}, V=\mathbb{C}\left[G / G_{N}\right]^{r}$ and $W=\operatorname{im}(A)_{G_{N}}$, and $V^{\prime}=A_{G_{N}}^{\prime} V_{1}$; then to $f=A_{G_{N}}, V=W=\operatorname{im}(A)_{G_{N}}$, $V^{\prime}=V_{2}$ and finally to $f=h_{G_{N}}, V=\mathbb{C}\left[G / G_{N}\right]^{r}, W=\operatorname{im}(A)_{G_{N}}$ and $V^{\prime}=V_{1}$. We conclude that

$$
\frac{\log \left(\operatorname{det}^{\prime}\left(A_{G_{N}}\right)\right)}{\left[G: G_{N}\right]}=\frac{\log \left(\operatorname{det}^{\prime}\left(h_{G_{N}}\right)^{-1} \operatorname{det}^{\prime}\left(A_{G_{N}}^{\prime}\right) \operatorname{det}^{\prime}\left(g_{G_{N}}\right)\right)}{\left[G: G_{N}\right]}+o(1)
$$

and we see that to prove the proposition it suffices to consider the case with nonzero determinant, since we can then apply it to the matrices $A^{\prime}, h h^{*}$ and $g g^{*}$.

We suppose now that $P=\operatorname{det}(A) \neq 0$; this case is proved as in Lück [17, Lemma 13.53]: we decompose $\mathbb{C}\left[G / G_{N}\right]^{n}$ as the sum $\bigoplus_{\zeta \in G_{N}^{\perp}} V_{\zeta}^{n}$ where $G / G_{N}$ acts on the line $V_{\zeta}$ through the character induced by $\zeta$ so that $A_{G_{N}}$ is represented on $V_{\zeta}^{n}$ by the matrix $A(\zeta)$. The number of $\zeta \in G_{N}^{\perp}$ with $\operatorname{det}(A)(\zeta)=0$ is $\leq \log \left[G: G_{N}\right]$ and since the singular values of the $A_{G_{N}}$ are bounded above by a constant an below by (2-6) we get

$$
\begin{aligned}
\frac{1}{\left[G: G_{N}\right]} \log \operatorname{det}^{\prime}\left(A_{G_{N}}\right) & =\frac{1}{\left[G: G_{N}\right]} \sum_{\zeta \in G_{N}^{\perp}} \log \operatorname{det}^{\prime} A(\zeta) \\
& \sim \frac{1}{\left[G: G_{N}\right]} \sum_{\zeta \in G_{N}^{\perp}, \operatorname{det}(A)(\zeta) \neq 0} \log \operatorname{det}(A(\zeta)) .
\end{aligned}
$$

Applying (i) of Proposition 2.7 to the right hand side we see that it converges to $\log \operatorname{det}_{\mathcal{N}(G)}(A)$ as $N \rightarrow \infty$.

Remark There is a general condition under which it is known that the approximation of the Fuglede-Kadison determinant is true (see Lück [16, Theorem 3.4]). We could have checked this condition directly for the matrices $A_{G_{N}}$ using (iii) of Proposition 2.7, but we have chosen to give a direct proof instead.

\subsection{Growth of volumes for $\ell^{2}$-acyclic complexes}

We treat here the growth of the parasite term $\prod_{i} \operatorname{vol}\left(H_{i}\right)^{(-1)^{i}}$ in (1-4). The following proposition is an adaptation of the main result of Bergeron-Venkatesh [1, section 7]. 
Proposition 2.9 Let $C_{*}$ be a finite complex of finitely generated $\mathbb{Z}[G]$-modules. Suppose that for some $i$ we have $H_{i}^{(2)}(C)=0$. Then for a sequence $G_{N}$ obtained from Proposition 2.7 we have

$$
\left|\log \operatorname{vol}\left(H_{i}\left(C_{G_{N}}\right)_{\text {free }}\right)\right|=O\left(\log \left[G: G_{N}\right]^{2}\right) .
$$

Proof For convenience we use the notation in [1] and denote $R_{i}(A)=\operatorname{vol}\left(H_{i}(A)_{\text {free }}\right)$ for a finite complex of free finitely generated $\mathbb{Z}$-modules $A_{*}$. The two next lemmas are proved there:

Lemma 2.10 (Bergeron-Venkatesh [1]) Let a finite group $K$ act by isometries on a finite complex $A_{*}$ of free $\mathbb{Z}$-modules endowed with metrics. Let $M$ be bigger than all singular values of the differentials of $A_{*}$ an suppose that the $A_{i}$ are generated by vectors with length less than $v$. Suppose that all irreducible characters of $K$ appearing in the $K$-vector space $H_{i}(A) \otimes \mathbb{C}$ are contained in a subset $X$ and denote by $D$ the maximal dimension of the $X$-isotypical component of the $A_{j}$. Then the following inequality holds:

$$
R_{i}(A) \geq\left(M v|K|^{5}\right)^{-D}
$$

Lemma 2.11 (Bergeron-Venkatesh [1]) Let $A$ be as above and $B_{*}$ be the dual complex $B_{n-j}=\operatorname{hom}\left(A_{j}, \mathbb{Z}\right)$ with the dual metric. We have

$$
R_{j}\left(A_{*}\right) R_{n-j}\left(B_{*}\right)=1 .
$$

Now we know that the differentials of the complexes $\left(C_{*}\right)_{H}$ have their singular values bounded by a constant $M$ depending only on $C_{*}$ and that the $\left(C_{j}\right)_{H}$ are generated by vectors of length one. Let $X$ be the set of irreducible characters of $G / H$ appearing in $H_{i}\left(C_{H}\right) \otimes \mathbb{C}$ and $D$ the maximal dimension of the $X$-isotypical component of the $\left(C_{j}\right)_{H}$. Lemma 2.10 yields

$$
R_{i}\left(C_{H}\right) \geq\left(M[G: H]^{5}\right)^{-D} .
$$

Now Lemma 2.11 implies that

$$
\left(M[G: H]^{5}\right)^{D} \geq R_{i}\left(C_{H}\right) \geq\left(M[G: H]^{5}\right)^{-D} .
$$

Since all $C_{j}$ are contained in $\mathbb{Z}[G]^{n}$ for some $n$ we see that for any set of characters $Y$ the dimension of the $Y$-isotypical component of $\left(C_{j}\right)_{H} \otimes \mathbb{C}$ is less than $n|Y|$. From 
Proposition 2.3 and (ii) of Proposition 2.7 we get that $D$ in (2-7) is an $O\left(\log \left[G: G_{N}\right]\right)$ and thus that

$$
\left|\log R_{i}\left(C_{G_{N}}\right)\right|=O\left(\log \left[G: G_{N}\right]^{2}\right) .
$$

Corollary Let $C_{*}$ be a finite $\ell^{2}$-acyclic complex of free finitely generated $\mathbb{Z}[G]-$ modules. There exists a sequence $G_{N}$ so that we have

$$
\lim _{N \rightarrow \infty}\left(\prod_{i}\left|H_{i}\left(C_{G_{N}}\right)_{\mathbb{Z}-\text { tors }}\right|^{(-1)^{i}}\right)^{\frac{1}{\left[G: G_{N}\right]}}=\tau^{(2)}(C) .
$$

Proof From Proposition 2.9 we get that we can choose $G_{N}$ so that for all $i$ we have

$$
\frac{\left|\log \operatorname{vol}\left(H_{i}\left(C_{G_{N}}\right)_{\text {free }}\right)\right|}{\left[G: G_{N}\right]}
$$

tends to 0 for all $i$. From Proposition 2.2 applied to the $d_{i}$ we get that we can choose $G_{N}$ so that furthermore

$$
\frac{\log \tau\left(C_{G_{N}}\right)}{\left[G: G_{N}\right]} \underset{N \rightarrow \infty}{\longrightarrow} \tau^{(2)}(C) .
$$

The corollary then follows from (1-4).

\section{Proof of the main theorems}

We recall notation from the Introduction: if $X$ is a CW-complex with universal covering $\tilde{X}$ and a surjection $\phi: \pi_{1}(M) \rightarrow G \cong \mathbb{Z}^{m}$, for any subgroup $H \subset G$ we denote by $X_{H}$ the Galois covering of $X$ given by $\phi^{-1}(H) \backslash \tilde{X}$; its Galois group is $G / H$. We also denote the free abelian covering of $X$ given by $\operatorname{ker}(\phi) \backslash \tilde{X}$ by $\widehat{X}$. Theorem 0.1 and Theorem 0.2 are immediate consequences of the following result:

Theorem 3.1 For all $i$, let $\Delta_{j}\left(H_{i}(\widehat{X})\right)$ be the first nonzero Alexander polynomial. There exists a sequence of subgroups $G_{N} \subset G$ with $\alpha\left(G_{N}\right) \underset{N \rightarrow \infty}{\longrightarrow} \infty$ such that

$$
\lim _{N \rightarrow \infty} \frac{\log \left|H_{i}\left(X_{G_{N}}\right)_{\text {tors }}\right|}{\left[G: G_{N}\right]}=m\left(\Delta_{j}\left(H_{i}(\widehat{X})\right)\right) .
$$

When $m=1$ we have

$$
\lim _{N \rightarrow \infty} \frac{\log \left|H_{i}\left(X_{N}\right)_{\text {tors }}\right|}{N}=m\left(\Delta_{j}\left(H_{i}(\widehat{X})\right)\right) .
$$

We begin by proving this for $m=1$, and then use Proposition 2.7 and the BombieriZannier theorem to deduce the result for $m>1$. 


\subsection{When $G$ is cyclic}

Let $M$ be a finitely generated $\mathbb{Z}\left[t^{ \pm 1}\right]$-module of rank $r$; put $M_{N}=M /\left(t^{N}-1\right) M$. We use the same notation for a finite complex of free modules $C_{*}, d_{*}$. We want to show that

$$
\lim _{N \rightarrow \infty} \frac{\log \left|H_{i}\left(C_{N}\right)_{\mathbb{Z}-\text { tors }}\right|}{N}=m\left(\Delta_{b_{i}^{(2)}(C)}\left(H_{i}(C)\right)\right)
$$

and also the corresponding limit for the module $M$

$$
\lim _{N \rightarrow \infty} \frac{\log \left|\left(M_{N}\right)_{\mathbb{Z}-\text { tors }}\right|}{N}=m\left(\Delta_{r}(M)\right) .
$$

We shall deduce (3-1) from (3-2). The latter is proved in three steps: first we consider torsion-free modules, then torsion modules and finally we combine those two to prove the general case.

Torsion-free modules Suppose that $M$ is torsion-free; we want to show that

$$
\frac{1}{N} \log \left|\left(M_{N}\right)_{\text {tors }}\right| \underset{N \rightarrow \infty}{\longrightarrow} 0 .
$$

We will in fact show that the torsion in $M_{N}$ is bounded independently of $N$. We can pick an embedding of $M$ into a free module $L \cong \mathbb{Z}[G]^{n}$. Put $T=L / M$; then the $\mathbb{Z}$-torsion part of $T$ has a finite exponent: the $\mathbb{Z}$-torsion part of $T$ is isomorphic to $M^{\prime} / M$ where $M^{\prime}=L \cap M \otimes \mathbb{Q}$. The submodule $M^{\prime}$ is finitely generated by $x_{1}, \ldots, x_{k} \in L$, and for each index $i$ there is an integer $n_{i}$ so that $n_{i} x_{i} \in M$. It follows that the exponent of $M^{\prime} / M$, and thus that of $T$, divides $n_{1} \ldots n_{k}$. We can use this to bound the exponent of $M_{N}$ :

Lemma 3.2 For all $N$ the exponent of $\left(M_{N}\right)_{\text {tors }}$ divides that of the $\mathbb{Z}$-torsion part of $T=L / M$.

Proof Suppose that $x \in M$ descends to an element of order exactly $n$ in $M_{N}$; this means that there exists $y \in M$, with $y \notin d M$ for any divisor $d>1$ of $n$, such that $n x=\left(t^{N}-1\right) y$. In the free module $L$ we see (by applying the Gauss lemma coordinate by coordinate) that $n$ must divide $y$. It follows that $n^{-1} y$ has order exactly $n$ in $L / M$.

For the torsion-free module $M$, it follows from the above Lemma that the $\mathbb{Z}$-torsion in $M_{N}$ is always $K$-torsion with a $K$ not depending on $N$. Now (3-3) follows from the following lemma. 
Lemma 3.3 Let $M$ be a finitely generated $\mathbb{Z}\left[t^{ \pm 1}\right]$-module (resp. $C_{*}$ a finite $\mathbb{Z}\left[t^{ \pm 1}\right]-$ complex) and $K$ a nonzero integer. Suppose that the $K$-torsion part of $M$ (resp. $H_{i}\left(C_{*}\right)$ ) is finite; then the $K$-torsion in $M_{N}$ (resp. $H_{i}\left(C_{N}\right)$ ) is bounded independently of $N$.

Proof Let $p_{1}^{k_{1}} \ldots p_{l}^{k_{l}}$ be the prime factorization of $K$. The $K$-torsion part of $M_{N}$ can be written as

$$
\left(M_{N}\right)_{K-\text { tors }} \cong \bigoplus_{i=1}^{l}\left(\bigoplus_{j=1}^{d_{i, N}} \mathbb{Z} / p^{\alpha_{i, j}} \mathbb{Z}\right)
$$

where $d_{i, N}=\operatorname{dim}\left(M_{N} \otimes \mathbb{F}_{p_{i}}\right)-\operatorname{rk}_{\mathbb{Z}}\left(M_{N}\right)$ and $\alpha_{i, j} \leq k_{i}$. It follows immediately that

$$
\left|\left(M_{N}\right)_{K-\text { tors }}\right| \leq \sum_{i=1}^{l} p_{i}^{k_{i}}\left(\operatorname{dim}\left(M_{N} \otimes \mathbb{F}_{p_{i}}\right)-\operatorname{dim}\left(M_{N} \otimes \mathbb{Q}\right)\right) .
$$

Thus, we need only prove that for $p=p_{1}, \ldots, p_{l}$ the difference

$$
\operatorname{dim}\left(M_{N} \otimes \mathbb{F}_{p}\right)-\operatorname{dim}\left(M_{N} \otimes \mathbb{Q}\right)
$$

is bounded. This can be seen by examining the proof of Linnell-Lück-Sauer [15, Theorem 0.2(i)] in our very special case; we give a short direct proof here. Note that the proof is very similar to that of Proposition 2.3.

Suppose that $M$ is torsion-free, let $r=\operatorname{rk}_{\mathbb{Z}\left[t^{ \pm 1}\right]}(M)$, and choose an embedding of $M$ into a free module $L$ of rank $r$. Since we know by Proposition 2.3 that $\operatorname{rk}_{\mathbb{Z}}\left(M_{N}\right)-r N$ is bounded, we need to show that $\operatorname{dim}\left(M_{N} \otimes \mathbb{F}_{p}\right)-r N$ is bounded. In the sequel we denote abusively $M, L$ the $\mathbb{F}_{p}$-modules $M, L \otimes \mathbb{F}_{p}$. There exists a $f \in \mathbb{F}_{p}[t]$ such that $f L \subset M$. We have $f_{N} L_{N} \subset M_{N} \subset L_{N}$; thus we only need to show that $\operatorname{dim}_{\mathbb{F}_{p}} \operatorname{ker}\left(f_{N}\right)$ is bounded. Suppose that $L=\mathbb{F}_{p}\left[t^{ \pm 1}\right]$; then we can identify $L_{N}$ with the polynomials in $\mathbb{F}_{p}[t]$ of degree less than $N$. Let $\ell=\operatorname{deg}(f)$. Then if $h \in \mathbb{F}_{p}[t]$ is nonzero with $\operatorname{deg}(h)<N-\ell$ the product $f h$ is still a polynomial of degree $<N$ and thus $f h$ is not zero in $L_{N}$. It follows that $f_{N}$ is injective on a subspace of dimension $N-\ell$, so that its kernel has dimension less than $\ell$. If $L \cong \mathbb{F}_{p}\left[t^{ \pm 1}\right]^{n}$ we can make the same argument coordinate by coordinate.

In general, according to the hypothesis on the $p$-torsion in $M$ we have $\operatorname{rk}_{\mathbb{F}_{p}\left[t^{ \pm 1}\right]} M \otimes$ $\mathbb{F}_{p}=r$. Thus, to deduce the general case from the torsion-free case we need only show that for a $\mathbb{F}_{p}\left[t^{ \pm 1}\right]$-torsion module $T$ the dimension $\operatorname{dim}\left(T_{N}\right)$ is bounded. This is easily deduced from the result above by choosing a surjection from some $\left(\mathbb{F}_{p}\left[t^{ \pm 1}\right] /(f)\right)^{k}$ to $T$.

The statement about homology is deduced from the statement for modules in the exact same way as in the proof of Proposition 2.3. 
Torsion modules Let $T$ be a finitely generated torsion module over $\mathbb{Z}\left[t^{ \pm 1}\right]$; we want to show that

$$
\lim _{N \rightarrow \infty} \frac{\log \left|\left(T_{N}\right)_{\text {tors }}\right|}{N}=m\left(\Delta_{0}(T)\right)
$$

We first return to the more general situation where $G \cong \mathbb{Z}^{m}$ to make a number of considerations.

Lemma 3.4 Let $T$ be a finitely generated $\mathbb{Z}[G]$-torsion module. Then there exists a finite resolution of $T$ by finitely generated free modules, which we write as

$$
0 \longrightarrow F_{m+2} \stackrel{\phi_{m+2}}{\longrightarrow} \cdots \stackrel{\phi_{2}}{\longrightarrow} F_{1} \stackrel{\phi_{1}}{\longrightarrow} T \longrightarrow 0
$$

The complex $F_{*}, \phi_{*}$ is a $\ell^{2}$-acyclic complex and its $\ell^{2}$-torsion equals $\mathcal{M}\left(\Delta_{0}(T)\right)^{-1}$. Moreover, for all $i>1$ there exists finitely generated torsion-free modules $M_{i}$ such that for any subgroup $H \subset G$ the homology $H_{i}\left(F_{H}\right)$ embeds into $M_{i, H}$.

Proof The ring $\mathbb{Z}\left[t_{1}, \ldots, t_{m}\right]$ is a regular ring with dimension $m+1$ by Serre [23, IV.D, Proposition 25]; since its localizations are the same as those of $\mathbb{Z}\left[t_{1}^{ \pm 1}, \ldots, t_{m}^{ \pm 1}\right] \cong \mathbb{Z}[G]$ the latter is also regular, with the same dimension. Now this means that all finitely generated modules over $\mathbb{Z}[G]$ have a projective resolution of length $\leq m+2$. Since it is known that all projective $\mathbb{Z}[G]$-modules are free (see Lam [11, Corollary V.4.12]) we have in fact that this holds with a free resolution, so that we get (3-5). The complex $F_{*}$ is given by the sequence

$$
0 \rightarrow F_{m+2} \rightarrow \cdots \rightarrow F_{1} \rightarrow 0
$$

so that its homology is zero for $i>1$ and $T$ for $i=1$. Thus it is a finite $\ell^{2}$-acyclic complex of free finitely generated $\mathbb{Z}[G]$-modules, and Equation (1-5) allows to compute that its $\ell^{2}$ torsion equals $-m\left(\Delta_{0}(T)\right)$.

Since $\operatorname{im}\left(\phi_{i, H}\right)$ is equal to the image of $\operatorname{im}\left(\phi_{i}\right)_{H}=\operatorname{ker}\left(\phi_{i}\right)_{H}$, the homology $H_{i}\left(F_{H}\right)$ is equal to the quotient $\operatorname{ker}\left(\phi_{i, H}\right) / \operatorname{ker}\left(\phi_{i}\right)_{H}$. The map $\phi_{i}$ embeds $M_{i}=F_{i} / \operatorname{ker}\left(\phi_{i}\right)$ into $F_{i-1} ; \operatorname{ker}\left(\phi_{i, H}\right) / \operatorname{ker}\left(\phi_{i}\right)_{H}$ is isomorphic to the kernel of the induced map $M_{i, H} \rightarrow F_{i-1, H}$, which is of course embedded in $M_{i, H}$.

Now we return to $m=1$ : it follows from the above Lemma and (3-3) that for $i>1$ we have

$$
\frac{1}{N} \log \left|H_{i}\left(C_{N}\right)_{\text {tors }}\right| \leq \frac{1}{N} \log \left|\left(M_{i, N}\right)_{\text {tors }}\right| \underset{N \rightarrow \infty}{\longrightarrow} 0 .
$$

Thus (3-4) follows from the Corollary to Proposition 2.9 applied to the complex $F_{*}, \phi_{*}$ since $H_{1}\left(F_{N}\right) \cong T_{N}$. 
Conclusion Now we can prove (3-2): we have the exact sequence

$$
0 \rightarrow M_{\mathrm{tors}} \stackrel{i}{\rightarrow} M \rightarrow M^{\prime} \rightarrow 0
$$

where $M^{\prime}=M / M_{\text {tors }}$ is torsion free. Tensoring with $\mathbb{Z}[\mathbb{Z} / N \mathbb{Z}]$ yields

$$
\left(M_{\text {tors }}\right)_{N} \stackrel{i_{N}}{\rightarrow} M_{N} \rightarrow M_{N}^{\prime} \rightarrow 0 .
$$

Lemma 3.5 The map $i_{N}:\left(M_{\text {tors }}\right)_{N} \rightarrow M_{N}$ is injective for all $N$.

Proof Suppose that $x \in M_{\text {tors }}$ and the image of $x$ in $\left(M_{\text {tors }}\right)_{N}$ is in the kernel of $i_{N}$; this means that there exists a $y \in M$ such that $x=\left(t^{N}-1\right) y$. But it follows from this last equality that $y \in M_{\text {tors }}$, so that $x$ is already zero in $\left(M_{\text {tors }}\right)_{N}$.

Thus, for the module $M$ we have the inequality

$$
1 \leq \frac{\left|\left(M_{N}\right)_{\mathbb{Z}-\text { tors }}\right|}{\left.\mid\left(M_{\text {tors }}\right)_{N}\right)_{\mathbb{Z}-\text { tors }} \mid} \leq\left(M_{N}^{\prime}\right)_{\mathbb{Z}-\text { tors }}
$$

Since we have already proved the term on the right is bounded it follows that

$$
\begin{aligned}
\lim _{N \rightarrow \infty} \frac{1}{N} \log \left|\left(M_{N}\right)_{\mathbb{Z}-\text { tors }}\right| & =\lim _{N \rightarrow \infty} \frac{1}{N} \log \left|\left(\left(M_{\text {tors }}\right)_{N}\right)_{\mathbb{Z}-\text { tors }}\right| \\
& =m\left(\Delta_{0}\left(M_{\text {tors }}\right)\right) \\
& =m\left(\Delta_{r}(M)\right)
\end{aligned}
$$

which finishes the proof of (3-2).

If $C_{*}, d_{*}$ is a finite complex of free finitely generated $\mathbb{Z}\left[t^{ \pm 1}\right]$-modules the map from $\operatorname{ker}\left(d_{i}\right)_{N}$ to $\operatorname{ker}\left(d_{i, N}\right)$ induces a map $j_{N}: H_{i}(C)_{N} \rightarrow H_{i}\left(C_{N}\right)$. The same proof as that of Lemma $3.5^{1}$ yields that $j_{N}$ is injective so that we get

$$
1 \leq \frac{\left|H_{i}\left(C_{N}\right)_{\text {tors }}\right|}{\left|\left(H_{i}(C)_{N}\right)_{\text {tors }}\right|} \leq\left|\operatorname{coker}\left(j_{N}\right)_{\text {tors }}\right| .
$$

To deal with the right-hand side we use the same trick as to prove (3-4): the cokernel of $j_{N}$ embeds into $\operatorname{ker}\left(d_{i, N}\right) / \operatorname{ker}\left(d_{i}\right)_{N}$, which embeds into $M_{N}$ where $M=C_{i} / \operatorname{ker}\left(d_{i}\right)$ is torsion-free, so that $\left|\operatorname{coker}\left(j_{N}\right)_{\text {tors }}\right|$ is bounded. Summing up, we get that

$$
\lim _{N \rightarrow \infty} \frac{1}{N}\left|H_{i}\left(C_{N}\right)_{\text {tors }}\right|=\lim _{N \rightarrow \infty} \frac{1}{N}\left|\left(H_{i}(C)_{N}\right)_{\text {tors }}\right|=m\left(\Delta_{b_{i}^{(2)}(C)}\left(H_{i}(C)\right)\right)
$$

where the last equality follows from (3-2) applied to $H_{i}(C)$.

${ }^{1}$ Suppose that $x \in \operatorname{ker}\left(d_{i}\right)$ is zero in $H_{i}\left(C_{N}\right)$, that is, $x=y+\left(t^{N}-1\right) z, y \in \operatorname{im}\left(d_{i+1}\right), z \in\left(t^{N}-1\right) C_{i}$. We get that $\left(t^{N}-1\right) d_{i}(z)=0$, so that in fact $z \in \operatorname{ker}\left(d_{i}\right)$ and $x$ is already zero in $H_{i}(C)_{N}$. 


\subsection{Torsion-free modules, $m>1$}

Our aim here is to prove the equivalent of (3-3) for some sequence $G_{N}$ obtained from Proposition 2.7. Recall from the proof of Proposition 2.7 that we have defined, when $m>1$,

$$
G_{N}=r_{N}^{*} \oplus \mathbb{Z} k_{N} v_{N}
$$

where $r_{N}, v_{N} \in G$ so that the scalar product $\left(r_{N}, v_{N}\right)$ is equal to 1 and $\alpha\left(r_{N}^{*}\right)$ tends to infinity as $N$ does. By taking $k_{N}$ large enough we can ensure that this sequence satisfies the conclusions (i) and (ii) of Proposition 2.2, for any given finite collection of matrices. We want to show that given a torsion-free $\mathbb{Z}[G]$-module $M$ we can also choose $k_{N}$ so that

$$
\frac{1}{\left[G: G_{N}\right]} \log \left|\left(M_{G_{N}}\right)_{\text {tors }}\right| \underset{N \rightarrow \infty}{\longrightarrow} 0
$$

holds. We remark that $M_{r_{N}^{*}}$ is a $\mathbb{Z}\left[t^{ \pm v_{N}}\right]$ module (for $v \in \mathbb{Z}^{m}$ we use the notation $t^{v}$ to denote $\left.t_{1}^{v_{1}} \ldots t_{m}^{v_{m}}\right)$. The strategy is then to apply the results of the preceding section to the $\mathbb{Z}\left[t^{ \pm v_{N}}\right]$-modules $M \otimes \mathbb{Z}\left[G / r_{N}^{*}\right]$ together with the following result, the proof of which is postponed until Appendix A.

Proposition 3.6 Let $M$ be a $\mathbb{Q}[G]$-module embedded in a free module $L$; then for $N$ big enough there exists a product of cyclotomic polynomials $\Phi_{N}$ such that the kernel of $M_{r_{N}^{*}} \rightarrow L_{r_{n}^{*}}$ is annihilated by $\Phi_{N}\left(t^{ \pm v_{N}}\right)$.

Let $M$ be a torsion-free $\mathbb{Z}[G]$-module which we embed into a free module $L$. It follows from the above Proposition that for $N$ big enough the kernel $T$ of the map $M_{r_{N}^{*}} \rightarrow L_{r_{N}^{*}}$ is annihilated by $K \Phi_{N}$ where $K$ is the exponent of the $\mathbb{Z}$-torsion in $L / M$ and $\Phi_{N}$ is a product of cyclotomic polynomials in the variable $t^{v_{N}}$. The submodule $T$ is the $\mathbb{Z}\left[t^{ \pm v_{N}}\right]$-torsion part of $M_{r_{N}^{*}}$, so that the maps from $T_{N}=T /\left(t^{k_{N} v_{N}}-1\right) T$ to $M_{G_{N}}$ is injective according to Lemma 3.5. Let $M^{\prime}$ be the image of $M_{r_{N}^{*}}$ in $L_{r_{N}^{*}}$, and put $M_{N}^{\prime}=M^{\prime} /\left(t^{k_{N} v_{N}}-1\right) M^{\prime}$; it follows from the exact sequence

$$
0 \rightarrow T_{N} \rightarrow M_{G_{N}} \rightarrow M_{N}^{\prime} \rightarrow 0
$$

that the $\mathbb{Z}$-torsion in $M_{G_{N}}$ is less than the product $\left|\left(T_{N}\right)_{\text {tors }}\right| \times\left|\left(M_{N}^{\prime}\right)_{\text {tors }}\right|$. The $\mathbb{Z}\left[t^{ \pm v_{N}}\right]$-module $T^{\prime}=K T$ is annihilated by the polynomial $\Phi_{N}$, so that the order of $\left(T_{k}^{\prime}\right)_{\text {tors }}$ is an $o(k)$ according to (3-4). The torsion in $T_{N}$ is less than the $K$-torsion times the torsion in $T_{k_{N}}^{\prime}$, and the former is bounded (in $k$ ) according to Lemma 3.3; it follows that we can choose $k_{N}$ so that $\left|\left(T_{N}\right)_{\text {tors }}\right|<k_{N} / N$ (say). Since $M_{N}^{\prime}$ is $\mathbb{Z}\left[t^{ \pm v_{N}}\right]$-torsion-free (3-3) allows us to choose $k_{N}$ so that $\left|\left(M_{N}^{\prime}\right)_{\text {tors }}\right|<k_{N} / N$. The conclusion (3-7) follows (recall that $k_{N}=\left[G: G_{N}\right]$ ). 


\subsection{Conclusion}

We are now in position to prove the following theorem, from which Theorem 3.1 follows immediately.

Theorem $3.7 \quad$ (i) Let $M$ be a finitely generated module of rank $r$; then

$$
\lim _{N \rightarrow \infty} \frac{\log \left|\left(M_{G_{N}}\right)_{\text {tors }}\right|}{\left[G: G_{N}\right]}=m\left(\Delta_{r}(M)\right) .
$$

(ii) Let $C_{*}, d_{*}$ be a complex of finitely generated free modules, $r=\operatorname{rk} H_{i}(C)$; we have

$$
\lim _{N \rightarrow \infty} \frac{\log \left|H_{i}\left(C_{G_{N}}\right)_{\text {tors }}\right|}{\left[G: G_{N}\right]}=m\left(\Delta_{r}\left(H_{i}(C)\right)\right)
$$

Proof We use the same scheme of proof as in the cyclic case: we begin by proving (3-8) for a torsion module, then deduce the case of a finitely generated module, and finally use it to prove (3-9).

Modules First of all, the proof of (3-8) does not change from the case $m=1$ : Lemma 3.4, the corollary to Proposition 2.9 and (3-7) together imply that for a torsion module $T$ we can choose $k_{N}$ in (3-6) so that

$$
\frac{1}{\left[G: G_{N}\right]} \log \left|\left(T_{G_{N}}\right)_{\text {tors }}\right| \underset{N \rightarrow \infty}{\longrightarrow} m\left(\Delta_{0}(T)\right) .
$$

Now let $M$ be any finitely generated $\mathbb{Z}[G]$-module; we have an exact sequence

$$
0 \rightarrow M_{\text {tors }} \rightarrow M \rightarrow M^{\prime} \rightarrow 0
$$

where $M^{\prime}=M / M_{\text {tors }}$. According to (3-7) we can choose the $k_{N}$ so that $M_{G_{N}}^{\prime}$ has a negligible torsion, so that to deduce (3-8) from (3-10) we need only show that

$$
\frac{\log \left|\operatorname{ker}\left(\left(M_{\text {tors }}\right)_{G_{N}} \rightarrow M_{G_{N}}\right)_{\mathbb{Z}-\text { tors }}\right|}{\left[G: G_{N}\right]} \underset{N \rightarrow \infty}{\longrightarrow} 0 .
$$

The following result is deduced from Proposition 3.6 in Appendix A.

Proposition 3.8 For $N$ big enough the kernel of the map $\left(M_{\mathrm{tors}}\right)_{r_{N}^{*}} \rightarrow M_{r_{N}^{*}}$ is annihilated by $K \Phi_{N}$ where $\Phi_{N}$ is a cyclotomic polynomial and $K$ an integer (not depending on $N$ ). 
We use this and an argument similar to that used to prove Lemma 3.5 to deduce (3-11). Suppose that $x \in M_{\text {tors }}$ descends to an element in $\operatorname{ker}\left(\left(M_{\text {tors }}\right)_{G_{N}} \rightarrow M_{G_{N}}\right)$; then there exists $y \in M, z \in\left(r_{N}^{*}-1\right) M$ such that $x=\left(t^{k_{N} v_{N}}-1\right) y+z$. It follows that $y \in M_{\text {tors }}+\left(r_{N}^{*}-1\right) M$, so that we can write $x=\left(t^{k_{N} v_{N}}-1\right) y^{\prime}+z^{\prime}$ where $y^{\prime} \in M_{\text {tors }}$ and $z^{\prime} \in\left(r_{N}^{*}-1\right) M$ It follows that $x$ and $z^{\prime}$ have the same image in $\left(M_{\text {tors }}\right)_{G_{N}}$, so that the kernel $\operatorname{ker}\left(\left(M_{\mathrm{tors}}\right)_{G_{N}} \rightarrow M_{G_{N}}\right)$ is the image of $T=\operatorname{ker}\left(\left(M_{\mathrm{tors}}\right)_{r_{N}^{*}} \rightarrow M_{r_{N}^{*}}\right)$. According to Proposition 3.8, 3.3 and (3-4) we can choose $k_{N}$ so that $\left(T_{k_{N}}\right)_{\text {tors }}$ has order less than $k_{N} / N$. This implies that the torsion in the kernel of the map $\left(M_{\text {tors }}\right)_{G_{N}} \rightarrow M_{G_{N}}$ also has order less than $k_{N} / N$ and (3-11) follows.

Complexes Let $N$ be fixed. We now compare the growth of the $\mathbb{Z}$-torsion of $H_{i}(C)_{r_{N}^{*}} /\left(t^{k v_{N}}-1\right) H_{i}(C)_{r_{N}^{*}}$ and $H_{i}\left(C_{r_{N}^{*}} /\left(t^{k v_{N}}-1\right) C_{r_{N}^{*}}\right)$; more precisely we show that

$$
\begin{aligned}
\lim _{k \rightarrow \infty} \frac{1}{k}\left(\log \left|\left(H_{i}(C)_{r_{N}^{*}} /\left(t^{k v_{N}}-1\right) H_{i}(C)_{r_{N}^{*}}\right)_{\mathbb{Z}-\text { tors }}\right|\right. & \\
& \left.-\log \left|\left(H_{i}\left(C_{r_{N}^{*}} /\left(t^{k v_{N}}-1\right) C_{r_{N}^{*}}\right)\right)_{\mathbb{Z}-\text { tors }}\right|\right)=0
\end{aligned}
$$

Let $\phi$ denote the map from $\left(H_{i}(C)_{r_{N}^{*}}\right)_{\text {tors }}$ to $H_{i}\left(C_{r_{N}^{*}}\right)_{\text {tors }}$; we prove that there exists a cyclotomic polynomial $\Phi_{N}$ which annihilates $\operatorname{ker}(\phi)$. Let $x \in \operatorname{ker}\left(d_{i}\right)$ map to an element in $\operatorname{ker}(\phi)$; we can write $x=y+z$ where $z \in \operatorname{ker}\left(d_{i}\right) \cap\left(r_{N}^{*}-1\right) C_{i}$. By Proposition 3.6, since there is no $\mathbb{Z}$-torsion in $C_{i} / \operatorname{ker}\left(d_{i}\right)$, we get that there exists $\Phi_{N}$ such that $\Phi_{N} z \in\left(r_{N}^{*}-1\right) \operatorname{ker}\left(d_{i}\right)$, so that $\Phi_{N} x$ maps to 0 in $\left(H_{i}(C)_{r_{N}^{*}}\right)_{\text {tors }}$. As for coker $(\phi)$, applying Proposition 3.6 to the embedding of $C_{i} / \operatorname{ker}\left(d_{i}\right)$ into $C_{i-1}$ we get that there is an integer $K$ independent of $N$ and a cyclotomic polynomial $\Phi_{N}^{\prime}$ such that $K \Phi_{N}^{\prime} \operatorname{coker}(\phi)=0$. It follows from these remarks and (3-2), (3-1) that

$$
\begin{aligned}
0 \leq \lim _{k \rightarrow \infty} \frac{1}{k}\left(\log \left(H_{i}\left(C_{r_{N}^{*}} /\left(t^{k v_{N}}-1\right) C_{r_{N}^{*}}\right)\right)-\log \left(H_{i}(C)_{r_{N}^{*}} /\left(t^{k v_{N}}-1\right) H_{i}(C)_{r_{N}^{*}}\right)\right) \\
\leq \lim _{k \rightarrow \infty} \frac{1}{k} \log \left|\left(H_{i}\left(C_{r_{N}^{*}} /\left(t^{k v_{N}}-1\right) C_{r_{N}^{*}}\right)\right)_{K-\text { tors }}\right|
\end{aligned}
$$

It follows from Lemma 3.3 that the limit on the right is zero, proving (3-12). By choosing $k_{N}$ big enough we can thus suppose that

$$
\lim _{N \rightarrow \infty} \frac{\log \left|\left(H_{i}(C)_{G_{N}}\right)_{\mathbb{Z}-\text { tors }}\right|-\log \left|H_{i}\left(C_{G_{N}}\right)_{\mathbb{Z}-\text { tors }}\right|}{\left[G: G_{N}\right]}=0,
$$

so that we deduce (3-9) from (3-8) applied to $H_{i}(C)$. This completes the proof of Theorem 3.7. 


\section{Cyclic coverings of 3-manifolds}

In the case $m=1$, Theorem 3.1 yields that for any finite CW-complex $X$ with an infinite cyclic covering $\widehat{X}$ and $X_{N}=N \mathbb{Z} \backslash \widehat{X}$ we have

$$
\frac{\log \left|H_{i}\left(X_{N}\right)_{\text {tors }}\right|}{N} \underset{N \rightarrow \infty}{\longrightarrow} \Delta_{b_{i}^{(2)}(\widehat{X})}\left(H_{i}(\widehat{X})\right) .
$$

Letting $X$ be a cell structure on a compact n-manifold we get Theorem 0.2.

\subsection{A presentation for the Alexander module}

It would be nice to have an explicit formula for the Alexander polynomial $\Delta_{i}(\widehat{M})$. The simplest case is that of a manifold fibering over the circle, which is $\ell^{2}$-acyclic and thus already treated in Bergeron-Venkatesh [1, Corollary 7.7]: if $\varphi$ is the diffeomorphism gluing the fiber $S$ and $\varphi_{*}$ its action on $H_{1}(S)$ we have $\Delta_{0}(\widehat{M})=\operatorname{det}\left(1-t \varphi_{*}\right)$. In the general case, the covering $\widehat{M}$ is obtained from an epimorphism $\pi_{1}(M) \rightarrow \mathbb{Z}$, and all such morphisms are given by the intersection form with an embedded incompressible two-sided nonseparating surface in $M$ : see the proof of Hempel [8, Lemma 6.6]. From here on we suppose we have a closed 3-manifold $M$ containing an embedded incompressible two-sided nonseparating surface $S$. Let $\widehat{M}$ be the infinite cyclic covering of $M$ associated with $S$. Let $U \cong S \times(-1,1)$ be a tubular neighbourhood of $S$ in $M$ and let $M^{\prime}=M-U$. We denote by $S_{ \pm}$the connected components of $\partial M^{\prime}$ corresponding to $S \times\{ \pm 1\}$ in $\bar{U}$. The fundamental group of $M$ is then given by an HNN-extension: we consider $\pi_{1}\left(S_{ \pm}\right)$as subgroups of $\pi_{1}\left(M^{\prime}\right)$, and there is an isomorphism $\alpha: \pi_{1}\left(S_{+}\right) \rightarrow \pi_{1}\left(S_{-}\right)$such that

$$
\pi_{1}(M)=\left\langle\pi_{1}\left(M^{\prime}\right), t \mid \forall g \in \pi_{1}\left(S_{+}\right), \operatorname{tg}^{-1}=\alpha(g)\right\rangle .
$$

Let us denote by $\alpha_{*}$ the induced map $H_{1}\left(S_{+}\right) \rightarrow H_{1}\left(S_{-}\right)$. We were not able to get a formula as explicit as that above, but only to obtain a presentation of $H_{1}(\widehat{M})$.

We can give an explicit construction of $\widehat{M}$ using $S: \widehat{M}$ is diffeomorphic to the manifold obtained from $M^{\prime} \times \mathbb{Z}$ by identifying $S_{+} \times\{n\}$ with $S_{-} \times\{n+1\}$ using $\alpha$. Let $i$ be the embedding of $S$ in $M^{\prime}$ corresponding to $S_{+}$. We know that $H_{0}(\widehat{M})=0$ and the map from $H_{1}\left(S_{+}\right)$to $H_{1}\left(M^{\prime}\right)$ is injective; thus the homology long exact sequence coming from

$$
S \times \mathbb{Z} \underset{(1-t \alpha) i}{\longrightarrow} M^{\prime} \times \mathbb{Z} \rightarrow \widehat{M}
$$

yields the short exact sequence

$$
\mathbb{Z}\left[t^{ \pm 1}\right] \otimes H_{1}(S) \underset{\left(1-t \alpha_{*}\right) i_{*}}{\longrightarrow} \mathbb{Z}\left[t^{ \pm 1}\right] \otimes H_{1}\left(M^{\prime}\right) \rightarrow H_{1}(\widehat{M}) \rightarrow 0 .
$$


which is a presentation of $H_{1}(\widehat{M})$. However, in all generality it seems rather difficult to compute the minors of the matrix $\left(1-t \alpha_{*}\right) i_{*}$ due to the unknown nature of $i_{*}$.

\subsection{Examples}

Computation for rational homology cylinders Suppose that $M^{\prime}, i_{ \pm}$is a rational homology cylinder, that is, the homology map $i_{*}$ induces an isomorphism between rational homologies. Then we can compute the Alexander polynomial, generalising a result for knot complements:

Lemma 4.1 In the situation described above, $\widehat{M}$ is $\ell^{2}$-acyclic and $\Delta_{0}\left(H_{1}(\widehat{M})=\right.$ $\tau\left(M^{\prime}, S_{+}\right) \operatorname{det}\left(1-t \alpha_{*}\right)$ where $\tau\left(M^{\prime}, S_{+}\right)$denotes the order of $H_{1}\left(M^{\prime}\right) / i_{*} H_{1}(S)$.

Proof This is an immediate consequence of (4-1) since in this case the map (1-t $\left.t \alpha_{*}\right) \circ i_{*}$ is represented by a square matrix with nonzero determinant.

In the case where $M$ is a knot exterior we retrieve the result of Lickorish [14, Theorem 6.5]. Note also that by the Dehn-Nielsen theorem $\alpha$ corresponds to an element $\phi$ in the mapping class group of $S$ and we have $\operatorname{det}\left(1-t \alpha_{*}\right)=\operatorname{det}\left(1-t \phi_{*}\right)$, so that we also retrieve the case where $M$ is fibered.

Given an homology cylinder $M^{\prime}, i_{ \pm}$(that is, $i_{ \pm}$are embeddings of $S$ in $\partial M^{\prime}$ which induce an isomorphism in homology), Cha, Friedl and Kim [2, Section 4.3] construct by surgery on the interior another homology cylinder. Their construction does not affect the Alexander polynomial of the infinite cyclic covering of the 3-manifold $M=M^{\prime} /\left\{\forall x \in S, i_{+}(x)=i_{-}(x)\right\}$ dual to $S$.

Positive $\ell^{2}$-Betti number There indeed are cases where the infinite cyclic covering is not $\ell^{2}$-acyclic. A somewhat artificial example is obtained as follows: suppose that $M_{0}$ is a three-manifold having an infinite cyclic covering $\widehat{M}_{0}$ and that $N$ is another three-manifold with positive $b_{1}$. Then the connected sum $M=M_{0} \sharp N$ has an infinite cyclic covering $\widehat{M}$ diffeomorphic to $\widehat{M}_{0} \sharp\left(N \times \mathbb{Z}\right.$ ) (if $M_{0}^{\prime}$ is $M_{0}$ cut along a surface dual to the covering, it is obtained by attaching a copy of $N$ to each copy of $M_{0}^{\prime}$ in $\widehat{M}_{0}$ ). Thus we have

$$
H_{1}(\widehat{M}) \cong H_{1}\left(\widehat{M}_{0}\right) \oplus\left(\mathbb{Z}\left[t^{ \pm 1}\right] \otimes H_{1}(N)\right)
$$

and it follows that $b_{1}^{(2)}(\widehat{M}) \geq b_{1}(N)$. Note that we also have $\Delta_{b_{1}(N)+i}\left(H_{1}(\widehat{M})\right)=$ $\Delta_{i}\left(\widehat{M}_{0}\right)$.

A more interesting example is given by manifolds with large fundamental group. Recall that a group is said to be large when it has a finite-index subgroup surjecting onto 
a noncyclic free group. Suppose that $\pi_{1}(M)$ surjects onto the free product $\mathbb{Z} * \mathbb{Z}$; then we can take the surjection from $\pi_{1}(M)$ to $\mathbb{Z}$ obtained by projection onto the second free factor. Then the associated infinite cyclic covering $\widehat{M}$ has $b_{1}^{(2)}(\widehat{M}) \geq 1$. Indeed, the kernel of the map $\mathbb{Z} * \mathbb{Z} \rightarrow \mathbb{Z} / N \mathbb{Z}$ is the subgroup freely generated by $a^{N}, b, a b a^{-1}, \ldots, a^{N-1} b a^{1-N}$, so that $\pi_{1}\left(M_{N}\right)$ surjects onto a free group of rank $N$ and thus we have $b_{1}\left(M_{N}\right)=b_{1}\left(\pi_{1}\left(M_{N}\right)\right) \geq N$ (any three-manifold with infinite fundamental group is aspherical) so that $b_{1}^{(2)}(\widehat{M}) \geq 1$ by Lück's theorem.

Lots of arithmetic three-manifolds are known to have a large fundamental group, and it is expected that in fact all hyperbolic three-manifolds have a large $\pi_{1}{ }^{2}$. See for example Lackenby [10] for recent progress on this and its links with other conjectures in 3-manifold topology.

\section{Appendix A Loose ends}

We shall prove here the results used in the proof of Theorem 3.7. Recall that, for a subgroup $H \subset \mathbb{Z}^{m^{\prime}}$, we denote by $H^{*}$ the subgroup of vectors orthogonal to $H$ in $\mathbb{Z}^{m^{\prime}} ; \alpha(H)$ is the smallest length of a nonzero vector in $H$. We introduce notation we shall use throughout this Appendix: if $A$ is an $m \times m^{\prime}$ matrix with coefficients in $\mathbb{Z}$ we denote by $H_{A}$ the subgroup of $\mathbb{Z}^{m^{\prime}}$ spanned by its lines. We will continue to denote by $A$ the induced morphism from $\mathbb{Z}\left[\mathbb{Z}^{m^{\prime}}\right]$ to $\mathbb{Z}\left[\mathbb{Z}^{m}\right]$. Recall that we identify the group ring $\mathbb{Z}\left[\mathbb{Z}^{m^{\prime}}\right]$ with the Laurent polynomials $\mathbb{Z}\left[t_{1}^{ \pm 1}, \ldots, t_{m^{\prime}}^{ \pm 1}\right]$; we denote this identification by $v \mapsto t^{v}$.

\section{A.1 The Bombieri-Schinzel-Zannier theorem}

The following result is an immediate generalisation of a theorem by Bombieri and Zannier (improving on a previous result by Schinzel):

Theorem A.1 Let $f_{1}, \ldots, f_{k} \in \mathbb{Z}\left[\mathbb{Z}^{m^{\prime}}\right]$ be coprime polynomials. Then there exists a constant $c=c\left(f_{1}, \ldots, f_{m}\right)$ such that if the gcd of $A f_{1}, \ldots, A f_{k}$ is not equal to a cyclotomic polynomial then $\alpha\left(H_{A}^{*}\right) \leq c$.

Proof This follows from an easy induction using the theorem as stated in Schinzel [21, Theorem 45 and page 517]; see also Le [13, Proposition 5.2].

In the sequel we shall use the term "cyclotomic polynomial" to design multivariate generalised cyclotomic polynomials, that is, the evaluations of a cyclotomic polynomial at a monomial, as well as their finite products.

\footnotetext{
${ }^{2}$ Ian Agol recently announced that he had a proof of this conjecture, following a previous work of Dani Wise on the subject.
} 


\section{A.2 Proof of Proposition 3.6}

In the sequel we denote by $A^{n}$ the map induced by $A$ from $\mathbb{Z}\left[\mathbb{Z}^{m^{\prime}}\right]^{n}$, that is,

$$
A^{n}\left(f_{1}, \ldots, f_{n}\right)=\left(A f_{1}, \ldots, A f_{N}\right) .
$$

Proposition A.2 Let $M$ be a submodule in $\mathbb{Q}\left[\mathbb{Z}^{m^{\prime}}\right]^{n}$; there exists a constant $c_{M}$ such that for all $A$ with $\alpha\left(H_{A}^{*}\right)>c_{M}$, there exists a cyclotomic polynomial $\Phi_{A}$ so that

$$
\Phi_{A} \operatorname{ker}\left(A^{n}\right) \cap M \subset \operatorname{ker}(A) M .
$$

Proof In this proof we denote the group ring $\mathbb{Q}\left[\mathbb{Z}^{m^{\prime}}\right]$ by $R$, and $\mathbb{Q}\left[A \mathbb{Z}^{m^{\prime}}\right]$ by $R^{\prime}$. It suffices to prove the result when the image of $A$ is of rank one (that is, $A$ is a line matrix).

Suppose first that $M=f R$ is a nonzero principal ideal. For $\alpha(H)$ big enough the support of $f$ injects into $\mathbb{Z}^{m^{\prime}} / H$, in particular for $\alpha\left(H_{A}^{*}\right)$ big enough $A f \neq 0$. The ideal $\operatorname{ker}(A)$ is prime (because $R / \operatorname{ker}(A) \cong R^{\prime}$ is a domain), so that if $f g \in \operatorname{ker}(A)$ then $g \in \operatorname{ker}(A)$. This means that $f R \cap \operatorname{ker}(A)=f \operatorname{ker}(A)$.

We next take $M$ to be an ideal in $R$, and prove the result by induction on the number of generators. First we reduce to the case where $M$ is not contained in any principal ideal. Suppose that $f$ is the gcd of $M$; then we can write $M=f M^{\prime}$ where $M^{\prime}$ is not contained in any principal ideal. We have $\operatorname{ker}(A) \cap f M^{\prime}=f \operatorname{ker}(A) \cap f M^{\prime}$ by the principal case, so that if the result is true for the embedding of $M^{\prime}$ in $f R$ it is also true for $M$.

Thus, let $f_{1}, \ldots, f_{k}$ be a minimal generating family for $M$ with no common factor. Suppose that $\alpha\left(H_{A}^{*}\right)>c=c\left(f_{1}, \ldots, f_{k}\right)$ with $c$ the constant from Theorem A.1; then the gcd of $A f_{1}, \ldots, A f_{k}$ must be equal to a cyclotomic polynomial $\Phi \in A R$ (we identify it with its evaluation at the monomial $t^{v_{A}}$ where $v_{A}$ generates a supplementary for $H_{A}^{*}$ ). Let $h=\sum_{i} h_{i} f_{i} \in \operatorname{ker}(A) \cap M$; we get that

$$
-A h_{1} A f_{1}=\sum_{i \geq 2} A h_{i} A f_{i}
$$

and so the gcd of $A f_{2}, \ldots, A f_{k}$ divides $\Phi A h_{1}$. We can write this gcd as $\sum_{i \geq 2} A g_{i} A f_{i}$ for some $g_{i} \in R$ since $R^{\prime}$ is a principal ring (because $A \mathbb{Z}^{m^{\prime}}$ is a cyclic group). Thus we get $\Phi A h_{1}=\sum_{i \geq 2} A h_{i}^{\prime} A f_{i}$ for some $h_{i}^{\prime} \in R$. It follows that we have

$$
\Phi h_{1}=\sum_{i \geq 2} h_{i}^{\prime} f_{i}+h_{1}^{\prime}
$$


for some $h_{1}^{\prime} \in \operatorname{ker}(A)$, and from that

$$
\Phi h=\Phi h_{1}^{\prime} f_{1}+\sum_{i \geq 2}\left(h_{i}+h_{i}^{\prime} f_{1}\right) f_{i}
$$

Let $M^{\prime}=\left(f_{2}, \ldots, f_{k}\right)$. By the induction hypothesis, if $\alpha\left(H_{A}^{*}\right)>c_{M^{\prime}}$ then the sum $\sum_{i \geq 2}\left(h_{i}+h_{i}^{\prime} f_{1}\right) f_{i} \in \Phi^{\prime} \operatorname{ker}(A) M^{\prime}$, so we get the result with $c_{M}=\max \left(c, c_{M^{\prime}}\right)$.

Suppose now that $\operatorname{rk}(M)=1$ and $M$ is embedded in $R^{n}$; up to passing to a larger free module (in $R \otimes \mathbb{Q}\left(\mathbb{Z}^{m^{\prime}}\right)^{n}$ ) we may suppose that $M$ is contained in a copy of $R$ that is a direct factor of $R^{n}$; then we can apply the above arguments to this embedding of $M$ in $R$ and get the result.

Now let us prove the general result by induction on the rank. Let $M$ have rank $k>1$ and be embedded in $R^{n}$; let $l<n$ so that the intersection $M_{0}=M \cap\left(R^{l} \times 0\right)$ has rank one. Put $M_{1}=M / M_{0}$; this is torsion-free and we have a commutative diagram

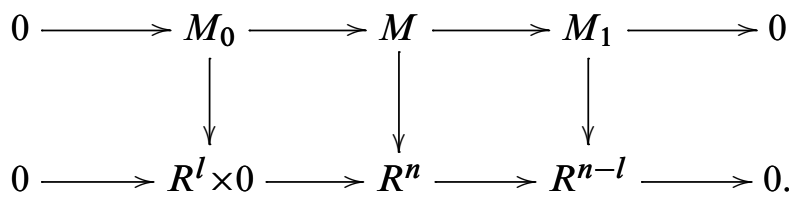

Suppose that $x \in \operatorname{ker}\left(A^{n}\right) \cap M$; its projection $x_{1}$ in $M_{1}$ lies in $\operatorname{ker}\left(A^{n-l}\right) \cap M_{1}$ and, by the induction hypothesis, there exists a cyclotomic $\Phi$ such that $\Phi x_{1} \in \operatorname{ker}(A) M_{1}$. It follows that $\Phi x=y+x_{0}$ for some $y \in \operatorname{ker}(A) M$ and $x_{0} \in \operatorname{ker}\left(A^{n}\right) \cap M_{0}$. By the induction hypothesis we get that there exists a cyclotomic $\Phi^{\prime}$ such that $\Phi^{\prime} x_{0} \in$ $\operatorname{ker}(A) M_{0}$. Finally, we have that $\Phi \Phi^{\prime} x \in \operatorname{ker}(A) M$.

Proof of Proposition 3.6 The $\operatorname{kernel} \operatorname{ker}(A)$ is easily seen to be equal to $\left(H_{A}^{*}-1\right) R$; indeed, the groups $A \mathbb{Z}^{m^{\prime}}$ and $\mathbb{Z}^{m^{\prime}} / H_{A}^{*}$ are isomorphic via $A$ so that the kernels of the maps $\mathbb{Z}\left[\mathbb{Z}^{m^{\prime}}\right] \rightarrow \mathbb{Z}\left[A \mathbb{Z}^{m^{\prime}}\right]$ and $\mathbb{Z}\left[\mathbb{Z}^{m^{\prime}}\right] \rightarrow \mathbb{Z}\left[\mathbb{Z}^{m^{\prime}} / H_{A}^{*}\right]$ must be equal. The first is $\operatorname{ker}(A)$ and the second $\left(H_{A}^{*}-1\right) \mathbb{Z}\left[\mathbb{Z}^{m^{\prime}}\right]$. Thus if $M \subset L$ is a submodule of a free module, the above Proposition yields that there exists a cyclotomic $\Phi_{A}$ such that $\Phi_{A}$ annihilates the kernel of the map

$$
M_{H_{A}^{*}}=M /\left(H_{A}^{*}-1\right) M \rightarrow L /\left(H_{A}^{*}-1\right)=L_{H_{A}^{*}} .
$$

Proposition 3.6 follows by taking $A$ to be the line matrix $r_{N}$ since $\alpha\left(r_{N}^{*}\right)$ tends to infinity. 


\section{A.3 Proof of Proposition 3.8}

We will in fact prove Proposition 3.8 for any sequence of subgroups of finite index which all contain a direct factor and whose smallest length tends to infinity. The method is to use induction on the rank. Here is the result that allows this:

Lemma A.3 Let $H$ be a subgroup of $\mathbb{Z}^{m^{\prime}}$ which contains a direct factor of $\mathbb{Z}^{m^{\prime}}$ of rank $m^{\prime}-1$, and such that $\alpha(G)>c$. Then there exists subgroups $H=H_{1} \supset H_{2} \supset$ $\ldots \supset H_{m^{\prime}}=0$ such that all inclusions are of corank one and all $H_{i}, i \geq 2$ are direct factors. Moreover, they all satisfy $\alpha\left(H_{i}^{*}\right) \geq c$.

Proof Let $\mathrm{H}_{2}$ be a direct factor contained in $H$. It has rank $m^{\prime}-1$, so the intersection $H_{2} \cap\left(0 \times \mathbb{Z}^{2}\right)$ is not empty. Let $v=(0, a, b)$ be in this intersection; we may suppose that $\operatorname{gcd}(a, b)=1$ since $H_{2}$ is a direct factor, and then we have $\min (|a|,|b|) \geq$ $\alpha(H)>c$. Thus we see that $\alpha\left(v^{*}\right)>c$. Now we only have to choose a basis $\left\{v_{1}=v, v_{2}, \ldots, v_{m^{\prime}-1}\right\}$ of $H$ and put $H_{i}=\sum_{j=1}^{i} \mathbb{Z} v_{i}$; the $H_{i}$ all contain $v$ and so $\alpha\left(H_{i}^{*}\right) \geq \alpha\left(v^{*}\right)>c$, and it is clear that they satisfy the other conclusions.

In consequence, Proposition 3.8 follows from:

Proposition A.4 Let $M$ be a finitely generated module over $R$. There exists a constant $c$ depending on $M$ such that the following holds. If $H$ is a subgroup of $\mathbb{Z}^{m^{\prime}}$ of corank $\geq 1$ having a sequence of subgroups $H_{2} \supset \ldots \supset H_{m^{\prime}}$ such that all inclusions are of corank one, all $H_{i}$ are direct factors and they all satisfy $\alpha\left(H_{i}^{*}\right)>c$, then the kernel of the map $\left(M_{\mathrm{tors}}\right)_{H} \rightarrow M_{H}$ is annihilated by a cyclotomic polynomial.

Proof If $H$ has rank one, we proved that the kernel is 0 in Lemma 3.5. We need the following result to carry out the induction step:

Lemma A.5 There is a constant $c$ so that for $\alpha\left(H_{A}^{*}\right)>c$ there exists a cyclotomic polynomial $\Phi$ such that the map $M_{R-\text { tors }} \rightarrow \Phi_{A}(A M)_{R^{\prime}-\text { tors }}$ is surjective.

Proof We put $M^{\prime}=M / M_{\text {tors }}$, embed $M^{\prime}$ in a free module $L$ and denote by $\pi$ the map $M \rightarrow L$ this yields. Suppose that $x \in M$ maps to a $R^{\prime}$-torsion element in $A M$; this means that there exists a $f \in R$ with $A f \neq 0$ such that $\pi(f x) \in \operatorname{ker}(A) \pi(M)$. Let $c$ be the constant for $M^{\prime} \subset L$ from Proposition 3.6; we get that for $\alpha\left(H_{A}^{*}\right)>c$ there exists a cyclotomic $\Phi$ such that $\Phi \pi(f x) \in \operatorname{ker}(A) M^{\prime}$. It follows that $\Phi x=y+x^{\prime}$ where $y \in M_{R-\text { tors }}=\operatorname{ker}(\pi)$ and $x^{\prime} \in \operatorname{ker}(A) M$, and thus that $A \Phi A x$ is in the image of $M_{R-\text { tors }}$ in $A M$. 
Write $H=\mathbb{Z} v \oplus H_{2}$ where $H_{2}$ is a direct factor of $\mathbb{Z}^{m^{\prime}}$. Let $x \in M_{\text {tors }}$, suppose that $x \in(H-1) M$. Then we can write $x=\left(t^{v}-1\right) x^{\prime}+z$ where $z \in\left(H_{2}-1\right) M$. Let $A$ be a matrix with $\operatorname{ker}(A)=H_{2}$ (this is possible since $H_{2}$ is a direct factor); we get that $A x=\left(t^{A v}-1\right) A x^{\prime}$, so that $x^{\prime}$ is $R^{\prime}$-torsion in $A M^{\prime}$ (we have to suppose that the annihilator of $x$ is not contained in $\left(H_{2}-1\right) R$, but this is true for $\alpha\left(H_{2}\right)$ big enough). By the Lemma above we get that $\Phi x^{\prime}=y+z^{\prime}$ where $z^{\prime} \in \operatorname{ker}(A) M=(H-1) M, y \in M_{\text {tors }}$. It follows that $\Phi x=\left(t^{v}-1\right) y+z+z^{\prime}$, and we get that $\Phi^{\prime}\left(z+z^{\prime}\right) \in(H-1) M_{\text {tors }}$ for some cyclotomic $\Phi^{\prime}$, by the induction hypothesis.

\section{Appendix B The $\ell^{2}$-volume and $\ell^{2}$-torsion}

We introduce here a new $\ell^{2}$-invariant, the aim of which is to be able to mimic the arguments leading to (1-4). In particular, we deduce (1-5) as a particular case.

\section{B.1 Definition}

We want to define a $\ell^{2}$-volume for submodules of $\mathbb{Z}[G]^{n}$ by analogy with the lattices in a finite-dimensional Hermitian space. It is quite natural to do this for free submodules: if $L \subset \mathbb{Z}[G]^{n}$ is free of rank $r$, then all morphisms $\mathbb{Z}[G]^{r} \rightarrow \mathbb{Z}[G]^{n}$ with image $L$ have the same Fuglede-Kadison determinant since if we fix one such morphism $f$, then any other one can be written $f \circ u$ for a change of basis $u$ in $\mathbb{Z}[G]^{r}$, and all change of basis morphisms for free $\mathbb{Z}[G]$-modules have a unit for determinant and thus their Fuglede-Kadison determinant is equal to one by equation (1-1). Thus, choosing a matrix $A \in M_{n, r}(\mathbb{Z}[G])$ with image $L$ we can put, as in the finite-dimensional case,

$$
\operatorname{vol}^{(2)}(L)=\operatorname{det}_{\mathcal{N}(G)}(A)=\mathcal{M}\left(\operatorname{det}\left(A^{*} A\right)\right) .
$$

When $M$ is a submodule of $\mathbb{Z}[G]^{n}$ with rank $r$, we can pick a free submodule of maximal rank (Lemma 1.2). The quotient $M / L$ is torsion by Lemma 1.3 , so it has nonzero first Alexander polynomial. In the finite dimensional case, if we have a finite index submodule $V^{\prime}$ of a module $V$ then for any metric on $M$ the equality $\operatorname{vol}(V)=\left[V: V^{\prime}\right] \operatorname{vol}\left(V^{\prime}\right)$ holds. Since we are interested in approximation problems the analogue of the index we shall consider for the maximal rank submodule $L \subset M$ is $\Delta_{0}(M / L)$ (this is justified by (3-10)). We put

$$
\operatorname{vol}^{(2)}(M)=\frac{\operatorname{vol}^{(2)}(L)}{\mathcal{M}\left(\Delta_{0}(M / L)\right)} .
$$

First of all, we have of course to check this is well-defined: 
Lemma B.1 The real number $\frac{\mathrm{vol}^{(2)}(L)}{\mathcal{M}\left(\Delta_{0}(M / L)\right)}$ does not depend on the free submodule of maximal rank $L \subset M$.

Proof Let $L_{1}, L_{2}$ be two free submodules of maximal rank in $M$. Then $L_{1} \cap L_{2}$ is a submodule of maximal rank, and thus contains a free submodule of maximal rank. Thus it is enough to prove that the $\ell^{2}$-volume is the same when computed for two free submodules of maximal rank $L \supset L^{\prime}$. Let $Q$ be the coordinate matrix of some basis of $L^{\prime}$ in a basis of $L$. We have

$$
\operatorname{vol}^{(2)}\left(L^{\prime}\right)=\operatorname{det}_{\mathcal{N}(G)}(Q) \operatorname{vol}^{(2)}(L)=\mathcal{M}\left(\Delta_{0}\left(L / L^{\prime}\right)\right) \operatorname{vol}^{(2)}(L) .
$$

On the other hand, we have a short exact sequence $0 \rightarrow L / L^{\prime} \rightarrow M / L^{\prime} \rightarrow M / L \rightarrow 0$ which gives

$$
\mathcal{M}\left(\Delta_{0}\left(M / L^{\prime}\right)\right)=\mathcal{M}\left(\Delta_{0}\left(L / L^{\prime}\right)\right) \mathcal{M}\left(\Delta_{0}(M / L)\right)
$$

(by multiplicativity of $\Delta_{0}$ and the Mahler measure). Thus

$$
\begin{aligned}
\frac{\operatorname{vol}^{(2)}\left(L^{\prime}\right)}{\mathcal{M}\left(\Delta_{0}\left(M / L^{\prime}\right)\right)} & =\frac{\operatorname{vol}^{(2)}(L)}{\mathcal{M}\left(\Delta_{0}\left(M / L^{\prime}\right)\right) \mathcal{M}\left(\Delta_{0}\left(L / L^{\prime}\right)\right)} \\
& =\frac{\operatorname{vol}^{(2)}(L)}{\mathcal{M}\left(\Delta_{0}(M / L)\right)} .
\end{aligned}
$$

This concludes the proof.

\section{B.2 Metric rank formula}

We can now state the $\ell^{2}$ metric rank formula; the proof is similar to that of the finite-dimensional case.

Lemma B.2 Let $M, M^{\prime}$ be $\mathbb{Z}[G]$-submodules of free modules and $f$ a morphism from $M$ to $M^{\prime}$; we have then that

$$
\operatorname{vol}^{(2)}(f(M))=\frac{\operatorname{det}_{\mathcal{N}(G)}(f) \operatorname{vol}^{(2)}(M)}{\operatorname{vol}^{(2)}(\operatorname{ker}(f))} .
$$

Proof Renormalizing by an homothety we may suppose vol ${ }^{(2)}(M)=1$. Let $L^{\prime} \subset$ $\operatorname{ker}(f)$ and $L^{\prime \prime} \subset \operatorname{ker}(f)^{\perp}$ be free submodules of maximal rank; the direct sum $L^{\prime} \oplus L^{\prime \prime}$ is then a free submodule of maximal rank in $M$. Put $T^{\prime}=\operatorname{ker}(f) / L^{\prime}$, $T^{\prime \prime}=\operatorname{im}(f) / f\left(L^{\prime \prime}\right) \cong M / \operatorname{ker}(f) \oplus L^{\prime \prime}$ and $T=M / L^{\prime} \oplus L^{\prime \prime}$. We get a short exact 
sequence $0 \rightarrow T^{\prime} \rightarrow T \rightarrow T^{\prime \prime} \rightarrow 0$, for example by applying the Snake Lemma to the diagram

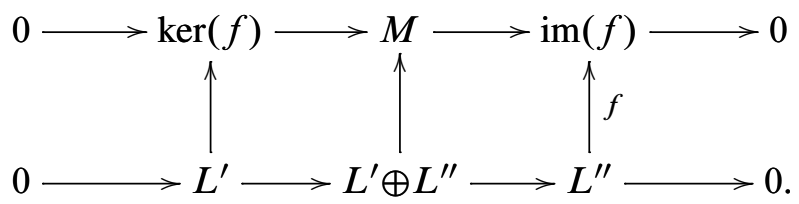

It follows that

$$
\mathcal{M}\left(\Delta_{0}(T)\right)=\mathcal{M}\left(\Delta_{0}\left(T^{\prime \prime}\right)\right) \mathcal{M}\left(\Delta_{0}\left(T^{\prime}\right)\right) .
$$

On the other hand, since $L^{\prime}$ and $L^{\prime \prime}$ are orthogonal we have

$$
\operatorname{vol}^{(2)}\left(L^{\prime}\right) \operatorname{vol}^{(2)}\left(L^{\prime \prime}\right)=\operatorname{vol}^{(2)}\left(L^{\prime}+L^{\prime \prime}\right) ;
$$

and since $M$ has unit volume this yields

$$
\begin{aligned}
\operatorname{vol}^{(2)}\left(L^{\prime}\right) \operatorname{vol}^{(2)}\left(L^{\prime \prime}\right) & =\mathcal{M}\left(\Delta_{0}(T)\right) \\
& =\mathcal{M}\left(\Delta_{0}\left(T^{\prime \prime}\right)\right) \mathcal{M}\left(\Delta_{0}\left(T^{\prime}\right)\right) .
\end{aligned}
$$

As $f_{\mid L^{\prime \prime}}$ is injective, $f\left(L^{\prime \prime}\right)$ is a free submodule of maximal rank in $\operatorname{im}(f)$ and thus

$$
\begin{aligned}
\operatorname{vol}^{(2)}(\operatorname{im}(f)) & =\operatorname{vol}^{(2)}\left(f\left(L^{\prime \prime}\right)\right) / \mathcal{M}\left(\Delta_{0}\left(\operatorname{im}(f) / f\left(L^{\prime \prime}\right)\right)\right) ; \\
& =\operatorname{vol}^{(2)}\left(f\left(L^{\prime \prime}\right)\right) / \mathcal{M}\left(\Delta_{0}\left(T^{\prime \prime}\right)\right) .
\end{aligned}
$$

Finally we have

$$
\operatorname{vol}^{(2)}\left(f\left(L^{\prime \prime}\right)\right)=\operatorname{det}_{\mathcal{N}(G)}(f) \operatorname{vol}^{(2)}\left(L^{\prime \prime}\right)
$$

From the three equalities above it follows that

$$
\begin{aligned}
\operatorname{vol}^{(2)}(\operatorname{im}(f)) & =\frac{\operatorname{det}_{\mathcal{N}(G)}(f) \operatorname{vol}^{(2)}\left(L^{\prime \prime}\right)}{\mathcal{M}\left(\Delta_{0}\left(T^{\prime \prime}\right)\right)} \\
& =\frac{\operatorname{det}_{\mathcal{N}(G)}(f) \mathcal{M}\left(\Delta_{0}\left(T^{\prime}\right)\right)}{\operatorname{vol}^{(2)}\left(L^{\prime}\right)} \\
& =\frac{\operatorname{det}_{\mathcal{N}(G)}(f)}{\operatorname{vol}^{(2)}(\operatorname{ker}(f))},
\end{aligned}
$$

and this concludes the proof of (B-1).

\section{B.3 Computation of the $\ell^{2}-$ torsion}

Let $C_{*}, d_{*}$ be a finite complex of free finitely generated $\mathbb{Z}[G]-$ modules. Let $L$ be a free maximal rank submodule in $H_{i}(C)$; then $L$ lifts to a free submodule $L^{\prime}$ in the 
orthogonal of $\operatorname{im}\left(d_{i+1}\right)$ in $\operatorname{ker}\left(d_{i}\right)$. Let $T$ be the quotient $\left(H_{i}(C) / H_{i}(C)_{\text {tors }}\right) / L$; we define

$$
\operatorname{vol}^{(2)}\left(H_{i}(C)\right)=\mathcal{M}\left(\Delta_{0}(T)\right) \operatorname{vol}^{(2)}\left(L^{\prime}\right) .
$$

In particular, when $C_{*}$ is $\ell^{2}$-acyclic this is equal to one since the orthogonal of $\operatorname{im}\left(d_{i+1}\right)$ in $\operatorname{ker}\left(d_{i}\right)$ is zero.

Lemma B.3 With notation as above, the following equality holds:

$$
\tau^{(2)}(C)=\prod_{i}\left(\frac{\mathcal{M}\left(\Delta_{b_{i}^{(2)}(C)}\left(H_{i}(C)\right)\right.}{\operatorname{vol}^{(2)}\left(H_{i}(C)\right)}\right)^{(-1)^{i}}
$$

Proof We denote by $M_{i}$ the orthogonal of $\operatorname{im}\left(d_{i+1}\right)$ in $\operatorname{ker}\left(d_{i}\right)$; this is a submodule of $C_{i}$ of rank $b_{i}^{(2)}(C)$. Let $L^{\prime}$ be a maximal rank free submodule in $\operatorname{im}\left(d_{i-1}\right)$ and $L^{\prime \prime}$ in $M_{i}$; put $L=L^{\prime} \oplus L^{\prime \prime}$, which is a free maximal rank submodule in $\operatorname{ker}\left(d_{i}\right)$. Since $M_{i}$ and $\operatorname{im}\left(d_{i-1}\right)$ are orthogonal we get $\operatorname{vol}^{(2)}(L)=\operatorname{vol}^{(2)}\left(L^{\prime}\right) \operatorname{vol}^{(2)}\left(L^{\prime \prime}\right)$. Putting $T=\operatorname{ker}\left(d_{i}\right) / L$ it follows that

$$
\operatorname{vol}^{(2)}\left(\operatorname{ker}\left(d_{i}\right)\right)=\frac{\operatorname{vol}^{(2)}(L)}{\mathcal{M}\left(\Delta_{0}(T)\right)}=\frac{\operatorname{vol}^{(2)}\left(L^{\prime}\right) \operatorname{vol}^{(2)}\left(L^{\prime \prime}\right)}{\mathcal{M}\left(\Delta_{0}(T)\right)} .
$$

Put $T^{\prime \prime}=M_{i} / L^{\prime \prime}$ and $T^{\prime}=\operatorname{im}\left(d_{i-1}\right) / L^{\prime}$; there is a natural injection of $T^{\prime} \oplus T^{\prime \prime}$ into $T$, whose cokernel is naturally isomorphic to $H_{i}(C) / M_{i}=: T_{0}$. Thus we get an exact sequence

$$
0 \rightarrow T^{\prime} \oplus T^{\prime \prime} \rightarrow T \rightarrow T_{0} \rightarrow 0
$$

and the equality

$$
\mathcal{M}\left(\Delta_{0}(T)\right)=\mathcal{M}\left(\Delta_{0}\left(T^{\prime}\right)\right) \mathcal{M}\left(\Delta_{0}\left(T^{\prime \prime}\right)\right) \mathcal{M}\left(\Delta_{0}\left(T_{0}\right)\right) .
$$

Finally, putting $T_{1}=\hat{H}_{i}(C) / M_{i}$ we get the exact sequence

$$
0 \rightarrow T_{1} \rightarrow T_{0} \rightarrow H_{i}(C)_{\text {tors }} \rightarrow 0
$$

and we can compute

$$
\mathcal{M}\left(\Delta_{0}\left(T_{0}\right)\right)=\mathcal{M}\left(\Delta_{0}\left(T_{1}\right)\right) \mathcal{M}\left(\Delta_{0}\left(H_{i}(C)_{\mathrm{tors}}\right)\right) .
$$


Putting the three equalities above together gives

$$
\begin{aligned}
\operatorname{vol}^{(2)}\left(\operatorname{ker}\left(d_{i}\right)\right) & =\frac{\operatorname{vol}^{(2)}\left(L^{\prime}\right)}{\mathcal{M}\left(\Delta_{0}\left(T^{\prime}\right)\right)} \times \frac{\operatorname{vol}^{(2)}\left(L^{\prime \prime}\right)}{\mathcal{M}\left(\Delta_{0}\left(T^{\prime \prime}\right)\right)} \times \frac{1}{\mathcal{M}\left(\Delta_{0}\left(T_{0}\right)\right)} \\
& =\operatorname{vol}^{(2)}\left(\operatorname{im}\left(d_{i-1}\right)\right) \times \frac{\operatorname{vol}^{(2)}\left(M_{i}\right)}{\mathcal{M}\left(\Delta_{0}\left(T_{1}\right)\right)} \times \frac{1}{\mathcal{M}\left(\Delta_{0}\left(H_{i}(C)_{\text {tors }}\right)\right)} \\
& =\frac{\operatorname{vol}^{(2)}\left(\operatorname{im}\left(d_{i-1}\right)\right) \operatorname{vol}^{(2)}\left(\hat{H}_{i}(C)\right)}{\mathcal{M}\left(\Delta_{0}\left(H_{i}(C)_{\text {tors }}\right)\right)} .
\end{aligned}
$$

From this and (B-1) it follows that

$$
\operatorname{vol}^{(2)}\left(\operatorname{ker}\left(d_{i}\right)\right)=\frac{\operatorname{det}_{\mathcal{N}(G)}\left(d_{i-1}\right) \operatorname{vol}^{(2)}\left(\hat{H}_{i}(C)\right)}{\mathcal{M}\left(\Delta_{0}\left(H_{i}(C)_{\text {tors }}\right)\right) \operatorname{vol}^{(2)}\left(\operatorname{ker} d_{i-1}\right)} .
$$

Taking the alternating product over $i$ we obtain

$$
1=\prod_{i}\left(\frac{\operatorname{det}_{\mathcal{N}(G)}\left(d_{i-1}\right) \operatorname{vol}^{(2)}\left(\hat{H}_{i}(C)\right)}{\mathcal{M}\left(\Delta_{0}\left(H_{i}(C)_{\mathrm{tors}}\right)\right)}\right)^{(-1)^{i}} .
$$

This completes the proof.

\section{References}

[1] N Bergeron, A Venkatesh, The asymptotic growth of torsion homology for arithmetic groups, to appear in J. Inst. Math. Jussieu arXiv:1004.1083

[2] J C Cha, S Friedl, T Kim, The cobordism group of homology cylinders, Compos. Math. 147 (2011) 914-942 MR2801405

[3] B Clair, K Whyte, Growth of Betti numbers, Topology 42 (2003) 1125-1142 MR1978050

[4] G Everest, T Ward, Heights of polynomials and entropy in algebraic dynamics, Universitext, Springer, London (1999) MR1700272

[5] F González-Acuña, H Short, Cyclic branched coverings of knots and homology spheres, Rev. Mat. Univ. Complut. Madrid 4 (1991) 97-120 MR1142552

[6] C M Gordon, Knots whose branched cyclic coverings have periodic homology, Trans. Amer. Math. Soc. 168 (1972) 357-370 MR0295327

[7] A Hatcher, Algebraic topology, Cambridge Univ. Press (2002) MR1867354

[8] J Hempel, 3-Manifolds, Ann. of Math. Studies 86, Princeton Univ. Press (1976) MR0415619

[9] E Kowalski, The large sieve and its applications, Cambridge Tracts in Mathematics 175, Cambridge Univ. Press (2008) MR2426239 
[10] M Lackenby, Finite covering spaces of 3-manifolds, from: "Proceedings of the International Congress of Mathematicians Volume II", Hindustan Book Agency, New Delhi (2010) 1042-1070 MR2827831

[11] T Y Lam, Serre's problem on projective modules, Springer Monographs in Mathematics, Springer, Berlin (2006) MR2235330

[12] W M Lawton, A problem of Boyd concerning geometric means of polynomials, $\mathbf{J}$. Number Theory 16 (1983) 356-362 MR707608

[13] T Le, Homology torsion growth and Mahler measure arXiv:1010.4199

[14] W B R Lickorish, An introduction to knot theory, Graduate Texts in Mathematics 175, Springer, New York (1997) MR1472978

[15] P Linnell, W Lück, R Sauer, The limit of $\mathbb{F}_{p}-$ Betti numbers of a tower of finite covers with amenable fundamental groups, Proc. Amer. Math. Soc. 139 (2011) 421-434 MR2736326

[16] W Lück, Approximating $L^{2}$-invariants by their finite-dimensional analogues, Geom. Funct. Anal. 4 (1994) 455-481 MR1280122

[17] W Lück, $L^{2}$-invariants: theory and applications to geometry and $K$-theory, Ergeb. Math. Grenzgeb. 44, Springer, Berlin (2002) MR1926649

[18] J P Mayberry, K Murasugi, Torsion-groups of abelian coverings of links, Trans. Amer. Math. Soc. 271 (1982) 143-173 MR648083

[19] J Porti, Mayberry-Murasugi's formula for links in homology 3-spheres, Proc. Amer. Math. Soc. 132 (2004) 3423-3431 MR2073320

[20] R Riley, Growth of order of homology of cyclic branched covers of knots, Bull. London Math. Soc. 22 (1990) 287-297 MR1041145

[21] A Schinzel, Polynomials with special regard to reducibility, Encyclopedia of Mathematics and its Applications 77, Cambridge Univ. Press (2000) MR1770638 With an appendix by Umberto Zannier

[22] K Schmidt, Dynamical systems of algebraic origin, Progress in Mathematics 128, Birkhäuser, Basel (1995) MR1345152

[23] J-P Serre, Local algebra, Springer Monographs in Mathematics, Springer, Berlin (2000) MR1771925 Translated from the French by CheeWhye Chin and revised by the author

[24] D S Silver, S G Williams, Mahler measure, links and homology growth, Topology 41 (2002) 979-991 MR1923995

[25] D S Silver, S G Williams, Torsion numbers of augmented groups with applications to knots and links, Enseign. Math. 48 (2002) 317-343 MR1955605

[26] D S Silver, S G Williams, Dynamics of twisted Alexander invariants, Topology Appl. 156 (2009) 2795-2811 MR2556037 
[27] V Turaev, Introduction to combinatorial torsions, Lectures in Mathematics ETH Zürich, Birkhäuser, Basel (2001) MR1809561 Notes taken by Felix Schlenk

Institut de mathématiques de Jussieu

4, place Jussieu, Case 247, 75252 Paris Cédex 5, France

raimbault@math.jussieu.fr

http://www.math.jussieu.fr/ raimbault/

Received: 8 April 2011 Revised: 24 February 2012 